\title{
Alkyl caffeates as antioxidants in O/W emulsions: Impact of emulsifier type and endogenous tocopherols
}

Sørensen, Ann-Dorit Moltke; Villeneuve, Pierre; Jacobsen, Charlotte

Published in:

European Journal of Lipid Science and Technology

Link to article, DOI:

10.1002/ejlt.201600276

Publication date:

2017

Document Version

Peer reviewed version

Link back to DTU Orbit

Citation (APA):

Sørensen, A-D. M., Villeneuve, P., \& Jacobsen, C. (2017). Alkyl caffeates as antioxidants in O/W emulsions: Impact of emulsifier type and endogenous tocopherols. European Journal of Lipid Science and Technology, 119(6), [e201600276]. https://doi.org/10.1002/ejlt.201600276

\section{General rights}

Copyright and moral rights for the publications made accessible in the public portal are retained by the authors and/or other copyright owners and it is a condition of accessing publications that users recognise and abide by the legal requirements associated with these rights.

- Users may download and print one copy of any publication from the public portal for the purpose of private study or research.

- You may not further distribute the material or use it for any profit-making activity or commercial gain

- You may freely distribute the URL identifying the publication in the public portal 


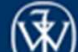

European Journal of

Lipid Science and Technology

\section{Alkyl caffeates as antioxidants in $0 / w$ emulsions: Impact of emulsifier type and endogenous tocopherols}

\begin{tabular}{|r|l|}
\hline Journal: & European Journal of Lipid Science and Technology \\
\hline Manuscript ID & ejlt.201600276.R1 \\
\hline Wiley - Manuscript type: & Research Article \\
\hline Date Submitted by the Author: & n/a \\
\hline Complete List of Authors: & $\begin{array}{l}\text { Sørensen, Ann-Dorit Moltke; Technical Univesity of Denmark, National } \\
\text { Food Institute } \\
\text { Villeneuve, Pierre; CIRAD, AMIS, UMR IATE } \\
\text { Jacobsen, Charlotte; Technical University of Denmark, National Food } \\
\text { Institute, Division of Industrial Food Research }\end{array}$ \\
\hline Keywords: & Caffeic acid, Lipid oxidation, Tween, Citrem, Phenolipids \\
\hline Additional Keywords (select & Emulsifiers, Emulsion, Fish oil \\
from list): & \multicolumn{2}{|c}{}
\end{tabular}


2 tocopherols

$4 \quad$ Ann-Dorit Moltke Sørensen ${ }^{1}$, Pierre Villeneuve ${ }^{2}$, Charlotte Jacobsen ${ }^{1}$

5

$6{ }^{1}$ Division of Food Technology, National Food Institute (DTU Food), Technical University of

7 Denmark, DK-2800 Kgs. Lyngby, Denmark

$8 \quad{ }^{2}$ CIRAD, UMR IATE, Montpellier, F-34398, France

$9 \quad{ }^{*}$ Corresponding author: adms@food.dtu.dk, Phone: +45 45252591

10

11 Running title: Effect of emulsifier and antioxidants on lipid oxidation

12 Keywords: Caffeic acid; Lipid oxidation; Tween; Citrem; Phenolipids; Fish oil; Antioxidant 13 interactions

15 Abbreviations: CA Caffeic acid; C1 Methyl caffeate; C4 Butyl caffeate; C8 Octyl caffeate; C12 16 Dodecyl caffeate; C16 Hexadecyl caffeate; C20 Eicosyl caffeate; CAT Conjugated autoxidizable 17 triene; CCL Critical chain length; FO Fish oil; FO/RO Fish oil and rapeseed oil mixture (1:1); PC 18 Principal component; PCA Principal component analysis; PV Peroxide value; THF Tetrahydrofuran 


\section{Abstract}

20

21

22

23

24

25

Antioxidant addition can be one strategy to limit lipid oxidation in emulsions. Research has proven that an important factor regarding the efficacy of antioxidants is their localization in the emulsion; however, other factors such as interactions with other components can also have an impact. Thus, the aim was to evaluate the impact of emulsifiers (Citrem and Tween80) and presence of endogenous tocopherols on the efficacies of caffeic acid and caffeates (C1-C20) as antioxidants in emulsions. Lipid oxidation was evaluated during storage and partitioning of caffeic acid and caffeates was estimated by measuring their concentrations in the aqueous phase.

Partitioning of caffeic acid and caffeates was influenced by emulsifier type and presence of endogenous tocopherols. Caffeic acid was the most efficient antioxidant in Citrem and Tween stabilized emulsions in the presence of endogenous tocopherol. In contrast, for Tween stabilized emulsions, caffeic acid acted as a prooxidant and the evaluated caffeates acted as strong antioxidants in the absence of endogenous tocopherol. Thus, when endogenous tocopherol was present lipophilization of caffeic acid did not increase its efficacy as an antioxidant. It is suggested that the differences observed in antioxidant efficiency with different emulsifiers and with and without endogenous tocopherols is due to emulsifier-antioxidant interactions and antioxidantantioxidant interactions in the emulsions.

Practical application: Food emulsions contain endogenous tocopherols, thus, the impact of endogenous tocopherols on the efficacy of applied antioxidants is of interest to the industry. So far the hypotheses about antioxidant in emulsions are based on simple emulsions systems without the presence of tocopherols. The finding in this study revealed that both emulsifier type and the presence of endogenous tocopherol had an impact on the efficacy of caffeic acid and caffeates due to emulsifier-antioxidant and antioxidant-antioxidant interactions. This highlights the importance of 
42 evaluating the antioxidant in each emulsion system before selecting antioxidants for optimal 43 protection against lipid oxidation.

44

45 
46

47

48

49

50

51

52

53

54

55

56

57

58

59

60

61

62

63

64

65

66

67

68

69

\section{Introduction}

An o/w emulsion consists of three different phases. A dispersed phase (oil) is present as droplets in a continuous phase (water) and separated by an interfacial region. Type of emulsions can range from very simple, when prepared from only a few ingredients, to more complex, when prepared with many different ingredients e.g. food emulsions. Lipid oxidation can occur rapidly in emulsions due to their large interfacial area. The interface region facilitates interactions between the lipids and water-soluble prooxidants [1].

Different strategies can be applied to limit lipid oxidation and thereby improve shelf life of emulsions [2]. One of them is addition of antioxidants; however, selection of the right antioxidant or mixture of antioxidants is difficult, since their efficacies are affected by the composition of the emulsions e.g. their localization and interaction with other components. An enormous amount of studies have been reported in the literature on antioxidants and their efficacies in model emulsions and more complex food emulsions. So far, two hypotheses about antioxidant efficacies in emulsions, namely the polar paradox hypothesis [3] and the cut-off effect [4] have been reported. In brief, the polar paradox hypothesizes that apolar antioxidants are more efficient in $\mathrm{O} / \mathrm{W}$ emulsions than polar antioxidants [3] due to differences in the antioxidants' affinity towards the different phases [5]. The cut-off effect can be seen as an extension of the polar paradox hypothesis, in which an optimal degree of lipophilisation for optimal antioxidant activity is observed [4], which is called critical chain length (CCL). The cut-off effect was observed from results obtained with chlorogenic acid and rosmarinic acid and their unbranched saturated alkyl esters (chlorogenates and rosmarinates). The efficacy of antioxidant homologues was related to the partitioning of these antioxidants in an emulsion system $[4,6]$. Based on these observations, it was assumed that when the lipophilized antioxidants had the CCL they were present in the highest concentration at the oilaqueous interface, where lipid oxidation is initiated. In addition, antioxidant homologues with chain 
length below and above CCL were driven away from the oil-aqueous interface [7]. These

antioxidant hypotheses are based on extensive research in simplified emulsions prepared with stripped oils, whereas food emulsions contain e.g. endogenous tocopherols. Only few compounds with different degree of lipophilization have been evaluated in several different emulsion systems. Caffeic acid and unbranched saturated caffeates have been evaluated both in a model emulsion (CAT assay) system [8], milk and mayonnaise [9] and CCL seemed to be influenced by the system. In addition, rosmarinic acid has also been evaluated in different systems; model emulsions and low moisture food (crackers). In model emulsions, a parabolic relationship between antioxidant efficacy and hydrophobicity was observed, with the intermediate polarity ( 8 carbon -18 carbon chain length) giving optimum activity $[6,10,11]$. In crackers, a linear relationship between antioxidant activity and hydrophobicity was observed [12].

Due to formerly obtained results with caffeic acid and caffeates in model emulsion, milk and mayonnaise $[8,9]$, it is hypothesized that emulsifier type and the presence of endogenous tocopherols can affect the partitioning of caffeic acid and caffeates and thereby change their efficacy in the emulsion systems. Hence, the aim of this study was to evaluate the impact of both emulsifier and presence of endogenous tocopherols on the efficacies of caffeic acid and caffeates (C1-C20). Two experiments were carried out as shown in Table 1. In the first experiment, the aim was to evaluate the effect of lipophilization of caffeic acid on its antioxidant efficacy in an $\mathrm{O} / \mathrm{W}$ emulsion prepared with unstripped oil and Citrem as emulsifier. The aim of the second experiment was to investigate whether the presence of endogenous tocopherol affected the optimal chain length of lipophilized caffeic acid when Tween was used as an emulsifier. Moreover, comparison of results of the two experiments enabled an evaluation of whether the emulsifier type, Citrem vs Tween affected the CCL in emulsions prepared with unstripped oil. 
93 In both experiments, the partitioning of the antioxidant was estimated by measuring its 94 concentration in the aqueous phase of the $\mathrm{O} / \mathrm{W}$ emulsion, a buffer/oil system and a buffer/emulsifier 95 system to evaluate if emulsifier type and the presence of endogenous tocopherols, affected the 96 partitioning of caffeic acid and caffeates in the emulsion system.

97 


\section{Material and Methods}

99

100

101

102

103

104

105

106

107

108

109

110

111

112

113

114

115

116

117

118

119

120

The oil used in this study was fish oil (FO) and a mixture between fish and rapeseed oil (FO/RO, 1:1). The two types of oil were supplied by Maritex A/S (TINE BA, Sortland, Norway). The quality of the FO was as follows: 0.3 meq. peroxides / $\mathrm{kg}$ oil, $250 \mathrm{mg} \alpha$-tocopherol $/ \mathrm{kg}, 98 \mathrm{mg} \gamma$ tocopherol / $\mathrm{kg}$ and $48 \mathrm{mg} \delta$-tocopherol $/ \mathrm{kg}$. Fatty acid composition of the fish oil was as follows: $14: 0,3.5 \% ; 16: 0,9.9 \% ; 16: 1 n-7,8.8 \% ; 18: 0,2.0 \% ; 18: 1 \mathrm{n}-9,16.3 \% ; 18: 1 \mathrm{n}-7,4.9 \% ; 18: 2 \mathrm{n}-6,1,8 \%$, $18: 3 n-3,2.6 \%, 18: 4 n-3,2.6 \%, 20: 1 n-7,12.6 \% ; 20: 5 n-3$ (EPA), 9.16\%; 22:1n-9, 5.8\%, 22:5n-3, $1.1 \%$ and $22: 6 n-3$ (DHA) $11.1 \%$. The total percentages of $n-3$ and $n-6$ PUFA in the FO were $24.0 \%$ and $1.8 \%$, respectively. The quality of the FO/RO was as follows: 0.3 meq. peroxides / $\mathrm{kg}$ oil, 230 $\mathrm{mg} \alpha$-tocopherol / kg, $31 \mathrm{mg} \beta$-tocopherol / kg, $151 \mathrm{mg} \gamma$-tocopherol / $\mathrm{kg}$ and $6 \mathrm{mg} \delta$-tocopherol / kg. The fatty acid composition of the FO/RO was as follows: 14:0, 1.7\%; 16:0, 7.1\%; 16:1 (n-7), $4.6 \% ; 18: 0,1.9 \% ; 18: 1$ (n-9), 38.3\%; 18:1 (n-7), 3.3\%; 18:2 (n-6), 10.5\%, 18:3 (n-3), 4.8\%; 18:4 (n-3), 1.2\%; 20:1, 6.9\%; 20:5 (n-3, EPA), 4.7\%; 22:1 (n-11), 3.0\% and 22:6 (n-3, DHA), 5.9\%. The total content of n-3 and n-6 PUFA in the FO/RO were $17.8 \%$ and $10.9 \%$, respectively.

The emulsifiers applied, Tween80 and Citrem LR 10 Extra (citric acid ester of mono- and diglyceride) without antioxidants were supplied by Sigma Aldrich (Steinheim, Germany) and Dupont (Danisco A/S, Grindsted, Denmark), respectively.

Alkyl caffeates were synthesized in an acid catalyzed reaction with caffeic acid and fatty alcohols with alcohol in excess as reaction medium or THF (tetrahydrofuran) as reaction medium. For further details refer to Sørensen et al. [8].

Tung oil (872 g/mol), Brij 35 (a nonionic polyoxyethylene surfactant, estimated Mw $1198 \mathrm{~g} / \mathrm{mol}$ ), phosphate buffer solution (PBS, pH 7.2), alumina, BHT (butylated hydroxytoluene), AAPH (2,2'Azobis(2-methylpropionamidine) dihydrochloride) and trolox were purchased from Sigma-Aldrich 
121 (Steinheim, Germany). Synperonic was purchased from CRODA (East Yorkshire, UK). All 122 solvents used were of HPLC grade and purchased from Lab-Scan (Dublin, Ireland). The external 123 standards used for quantification of secondary oxidation products were from Sigma Aldrich 124 (Steinheim, Germany).

125

126

\section{Experimental design}

127 For specific details on emulsifier, oil and antioxidants applied refer to Table 1. Experiment 1 was 128 designed to evaluate the effect caffeic acid and different alkyl caffeates as antioxidant in Citrem 129 stabilized emulsions in the presence of endogenous tocopherols due to the natural content of 130 tocopherols in oil. In this experiment fish oil was used, and a storage period of 15 days selected 131 based on previous storage experiment with fish oil.

132 Experiment 2 was designed to evaluate the influence of endogenous tocopherols on the efficacy of 133 caffeic acid and caffeates in Tween stabilized emulsions. Tween was selected as emulsifier, since 134 Citrem contains tocopherols. Emulsions were prepared with and without endogenous tocopherols. 135 Thus, the oil without endogenous tocopherols was stripped (removal of tocopherols). Stripping of 136 fish oil will increase oxidation rates tremendously. To slow down oxidation rate, a mixture of fish 137 and rapeseed oil was therefore used instead in this experiment. The storage time was selected based 138 on preliminary laboratory trials (data not shown).

139 Based on experiment 1 and 2 with endogenous tocopherols, the impact of emulsifier type is 140 evaluated despite different oil type and length of storage time.

142 Removal of tocopherols from oils 
$143 \mathrm{FO} / \mathrm{RO}$ was stripped from tocopherols using an alumina packed glass column using hexane. For 144 further details refer to Sørensen et al [8]. The stripped oil was bottled, flushed with nitrogen and 145 stored at $-80^{\circ} \mathrm{C}$ until use for production of emulsions. Furthermore, the absence of tocopherols in 146 the oil was checked by HPLC according to the AOCS method [13]. After oil stripping (removal of 147 tocopherols) the PV was 0.5 meq. peroxides / $\mathrm{kg}$ oil and tocopherols were not detected.

\section{Production of $\mathrm{O} / \mathrm{W}$ emulsions}

150

151

152

153

154

155

156

157

158

159

160

161

162

163

164

165

Both in experiment 1 and 2, the emulsion compositions were 5\% oil, 1\% emulsifier and 94\% 10 $\mathrm{mM}$ sodium acetate - imidazole buffer $(\mathrm{pH} 7)$. Antioxidants were diluted in methanol and added in concentrations of $100 \mu \mathrm{M}$. For the control emulsions (without antioxidant added), methanol was added in same amount as used for the methanolic antioxidant solutions added to the other emulsions. The short to medium chain phenolipids (C0 - C12) were added to the buffer (Citrem emulsions) and buffer-emulsifier mixture (Tween emulsions), whereas the long chain phenolipids (C16 - C20) were added to the oil-emulsifier mixture (Citrem emulsions) and oil (Tween emulsions) before the pre-homogenisation step.

Preparation of emulsions for storage experiment 1 were produced with pre-emulsification ( 2 min, Ultra-Turrax, Janke \& Kunkel IKA-Labortechnik, Staufen, Germany) followed by homogenization on a two-valve table homogenizer at a pressure of 800 bar (GEA Niro Soavi Spa, Parma, Italy). For further details refer to Sørensen et al. [14]. Production of emulsions for storage experiment 2 were pre-emulsified as in experiment 1 , but homogenized on a microfluidizer (9K, Microfluidics, Newton, MA, USA). Changes in the production between experiment 1 and 2 (homogenizer vs. microfluidizer) were done in order to operate with smaller emulsion volumes and thereby reduce the amount of phenolipids. Moreover, it became possible tocool the emulsion during production which 
166 was an advantage due to the fact that the oil was stripped from tocopherols and would therefore be 167 highly susceptible to oxidation during homogenization.

168 After production emulsions $(100 \mathrm{~g})$ were stored in $100 \mathrm{~mL}$ blue cap bottles at $20^{\circ} \mathrm{C} \pm 2^{\circ} \mathrm{C}$. Samples, 169 one bottle pr. code, were taken at specific time points and divided into brown glass bottles, flushed 170 with nitrogen and stored at $-40^{\circ} \mathrm{C}$ until analyses, except for samples used to determine droplet size, 171 these samples were measured at the sampling day without pre-freezing.

\section{Droplet size measurements}

173 Droplet size of the oil droplets in the $\mathrm{O} / \mathrm{W}$ emulsion was determined by laser diffraction 174 (Mastersizer2000, Malvern Instruments Ltd., Worcestershire, UK). Few droplets of the different 175 emulsions were suspended directly in recirculating water (2800 rpm, obscuration 12-14\%). Water $176\left(\mathrm{RI}_{\mathrm{water}}=1.330\right)$ and sunflower oil $\left(\mathrm{RI}_{\mathrm{oil}}=1.469\right)$ were used in this measurements as dispersant and 177 particle, respectively. Each sample was measured in triplicate $(n=3)$ and results are reported as 178 surface mean diameter, $\mathrm{D}_{3,2}[15]$.

\section{Lipid extractions from $\mathrm{O} / \mathrm{W}$ emulsions}

181 Peroxide value (PV) and tocopherol analyses are performed on lipid extracts. Thus, the lipids were 182 extracted from the emulsions prior to these analyses according to the method described by Bligh 183 and Dyer [16] using a reduced amount of solvent [17]. For each sample code two lipid extractions 184 were performed $(n=2)$.

\section{Tocopherols}


187 Lipid extracts were evaporated under nitrogen, re-dissolved in heptane and analyzed by HPLC 188 (Agilent 1100 Series, Agilent Technology, Palo Alto, CA, USA) according to the AOCS Official 189 Method Ce 8-89 [13]. A silica column (Waters (Dublin, Ireland), 150mm, 4.6mm, $3 \mu \mathrm{m}$ silica film) 190 was used for separation of the tocopherol homologues. This analysis was performed in duplicate on 191 each lipid extract and results reported as $\mu \mathrm{g}$ tocopherol / g emulsion.

192

193 Primary oxidation products: Peroxide value (PV)

\begin{abstract}
194 PVs in the lipid extracts were determined by colorimetric method based on formation of an iron195 thiocyanate complex. The colored complex was measured on a spectrophotometer at $500 \mathrm{~nm}$ 196 (Shimadzu UV1800, Shimadzu Scientific Instruments, Columbia, MD, USA) [18]. The analysis 197 was performed in duplicate and reported as meq peroxides / $\mathrm{kg}$ oil.
\end{abstract}

Secondary oxidation products: Volatiles

Volatiles were released from the $\mathrm{O} / \mathrm{W}$ emulsion using dynamic headspace $\left(45^{\circ} \mathrm{C}\right.$ for $30 \mathrm{~min}$, 201 nitrogen flow of $150 \mathrm{~mL} / \mathrm{min}$ ). Volatiles were then collected and trapped on Tenax GR packed 202 tubes. To avoid foam and thus water on the tubes, $4 \mathrm{~mL}$ of antifoam (Synperonic, conc. $8 \mathrm{~g} / \mathrm{L}$ 203 water) was added to each sample prior to the collection. Trapped volatiles were desorbed using an 204 automatic thermal desorber (ATD-400, Perkin Elmer, Waltham, MA, USA). The transfer line of 205 the ATD was connected to a gas chromatograph (Agilent 5890, Palo Alto, CA, USA) with a mass 206 selective detector (HP 5972). Volatiles were separated on a DB1701 column (30m x ID 0.25mm x $2071 \mu \mathrm{m}$ film thickness, J\&W Scientific, Folsom, CA, USA). The initial temperature of the oven was $20845^{\circ} \mathrm{C}$, which was kept for 5 minutes and then gradually increased as follows: $45-55^{\circ} \mathrm{C} 1.5^{\circ} \mathrm{C} / \mathrm{min}$, 
$20955-90^{\circ} \mathrm{C} 2.5^{\circ} \mathrm{C} / \mathrm{min}, 90-220^{\circ} \mathrm{C} 12^{\circ} \mathrm{C} / \mathrm{min}$ and kept at $220^{\circ} \mathrm{C}$ for 4 minutes. Calibration curves

210 prepared from external standards was used for quantification of different volatiles. In experiment 1

211 (Table 1), different concentrations of external standard solutions were prepared and $1 \mu \mathrm{L}$ was

212 placed in Tenax tubes and analyzed. In experiment 2 (Table 1), different concentrations of external

213 standard solutions were prepared and added to fresh emulsion without antioxidant. Volatiles were

214 collected in the same way as for samples. The 2,4-heptadienal external standard appears as two

215 peaks in the chromotogramme, these peaks are termed A and B. Each sample code was analyzed in

216 triplicate $(\mathrm{n}=3)$ and results reported as $\mathrm{ng}$ volatile / $\mathrm{g}$ emulsion.

218 Partitioning

219 Partitioning of caffeic acid and the different alkyl caffeates in buffer/oil, emulsifier/buffer and in $220 \mathrm{O} / \mathrm{W}$ emulsion was measured according to the method described by Schwarz et al. [19] with 221 modifications as described elsewhere. With this method it is assumed that the partitioning of the 222 antioxidants, equilibra reached, is not disrupted by centrifugation. For further details refer to 223 Sørensen et al. [20]. In short, the concentration of antioxidants was measured in the separated 224 aqueous phase of 3 different systems: buffer / oil (FO or FO/RO), buffer / emulsifier (Citrem or 225 Tween 80 ) and 5\% O/W emulsions. Antioxidants were dissolved in methanol and added in a 226 concentration of $100 \mu \mathrm{M}$. Separation of the aqueous phase was carried out $24 \mathrm{~h}$ after production of 227 the different systems. 
230 Caffeic acid and alkyl caffeates in the aqueous phases were analyzed by HPLC (Agilent 1100 231 Series, Agilent Technology, CA, USA) with a C18 Thermo Hypersil ${ }^{\circ}$ ODS $(250 \times 4.6$ mm, $5 \mu)$ 232 column and using a gradient elution at a flow rate of $1 \mathrm{~mL} / \mathrm{min}$. Solvent A was $3 \mathrm{mM}$ phosphoric 233 acid and solvent B was methanol. Gradient condition: 0-30 min 0-100\% B, 30-40 min 100\% B and $234 \quad 40-45 \min 100-0 \%$ B. Injection volume was $20 \mu \mathrm{L}$. Caffeic acid and different alkyl caffeates were 235 quantified by calibration curves of these compounds dissolved in methanol.

\section{Conjugated autoxidizable triene (CAT) Assay}

Stock solutions of the different compounds: caffeic acid, caffeates and trolox were prepared in methanol. The CAT assay was performed as described in Sørensen et al. [8] with a modification. This assay was performed with non-stripped tung oil, hence, the assay contained tocopherol and the evaluated antioxidant in a mixture. Each microplate well contained a microemulsion with the following composition: $115 \mu \mathrm{M}$ tung oil, $17 \mu \mathrm{M}$ Brij 35, $1 \mathrm{mM}$ AAPH and antioxidant (caffeic acid, caffeates or Trolox) in various concentrations. The progress of lipid oxidation was followed by measuring the decrease in absorbance at $273 \mathrm{~nm}$. Each antioxidant concentration was measured in triplicate on the plate and via independent measurements (two different microplates), $\mathrm{n}=6$. Results were expressed as CAT value (mean $\pm \mathrm{SD})$. This method was developed by Laguerre et al. [21]. For further details about the calculations refer to Laguerre et al. [4, 21].

\section{Data treatment}

250 Statistics. The results obtained were analyzed using one- and two-way ANOVA (GraphPad Prism, 251 Version 4.01, GraphPad Software Inc). Bonferroni multiple comparison post-test was used to 252 determine significant differences between samples or storage times. The significance level applied 
253 was $95 \%(p<0.05)$. Significant difference between samples is denoted with different superscripts 254 (i.e. a, b, c ect.).

255 Inhibition percentages. Since different oil and emulsifiers can influence amount of lipid oxidation, 256 inhibition percentages were used to compare the efficacy of the antioxidants in the different 257 emulsion systems. The antioxidants inhibition percentages were calculated according to the 258 following equation:

$$
\text { Inhibition }[\%]=\left(\frac{\text { Emulsion }_{\text {Control }}-\text { Emulsion }_{\text {Antioxidant }}}{\text { Emulsion }_{\text {Control }}}\right) \times 100
$$

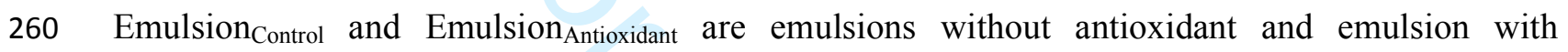
261 antioxidant added, respectively. The antioxidant has a prooxidative effect if the inhibition $<-5 \%$ 262 and an antioxidative effect $>5 \%$. The antioxidative effect can be categorized as weak $(5-20 \%)$, 263 intermediate $(20-50 \%)$ or strong $(>50 \%)$ effects [22].

Multivariate data analysis, Principal Component Analysis (PCA). Inhibition percentages

266 calculated from results obtained from PV, volatiles and tocopherol analysis were subjected to PCA 267 using Unscrambler version X10.3 (Camo, Oslo, Norway). The PCA model was built on inhibition 268 percentages calculated on the average of the measured data. Full cross validation was used to 269 validate the model. A PCA allows detection of similarities and dissimilarities between the different 270 samples in a score plot, whereas correlations between the measured variables are visualized in a 271 loadings plot. Connecting these plots in this case shows the degree of oxidation between the 272 different samples. 


\section{Results}

\section{$274 \quad$ Physical stability of the emulsions}

275 The physical stability of the emulsions was evaluated by following the oil droplet size (D3,2 276 (surface weighted mean)) over time. With Citrem as emulsifier (Exp. 1), the droplet size was 277 between $101-114 \mathrm{~nm}$ for the different Citrem stabilized emulsions (data not shown). The emulsion 278 prepared with Tween as emulsifier (Exp. 2), non-stripped FO/RO and stripped FO/RO had droplet 279 sizes between $124-131 \mathrm{~nm}$ and $126-131 \mathrm{~nm}$, respectively (data not shown). Emulsions produced 280 on the homogenizer (Exp. 1), resulted in slightly smaller oil droplets than emulsions produced on 281 the microfluidizer (Exp. 2). The small differences in oil droplet sizes, however significant, for 282 Citrem and Tween stabilized emulsions is suggested to be due to the different emulsification 283 equipment applied, the different emulsifiers used or a combination. Changes in droplet size during 284 storage were minor, but significant. The changes ranged from $-6 \mathrm{~nm}$ to $+2 \mathrm{~nm}$ in size. Since, the oil 285 droplets mainly had a slight decrease in size during storage, the changes is suggested to be due to 286 day to day instrumental variation. Hence, all emulsions were physically stable during the whole 287 storage time, 42 hours, 6 and 15 days, respectively.

\section{Partitioning of caffeic acid and caffeates}

290

291

292

293

294 295
The concentration of caffeic acid (CA) and caffeates (CA C1 - CA C12) were determined in the aqueous phase of three different systems to mimic the applied emulsion systems. Hence, the two different emulsifiers and non-stripped and stripped oil was applied. The results obtained are presented in Table 2. In general, the partitioning of caffeic acid and caffeates in the aqueous phase decreased with increased degree of lipophilization until chain length $\mathrm{C} 4$ after which the caffeates in most cases could not be detected in the aqueous phase. In the case of the buffer/Tween system 
296 octyl- (C8) and dodecyl caffeates (C12) were detected in the aqueous phase and the concentrations

297 were not significantly different from that of butyl caffeate (C4). The concentration of caffeic acid 298 and methyl caffeate in the aqueous phase was significantly lower when Tween was applied as 299 emulsifier than when Citrem was applied. Moreover, the concentration of butyl caffeate tended to 300 be lower (not significant) in the aqueous phase with Tween as emulsifier than with Citrem as also 301 observed for caffeic acid and methyl caffeate. These partitioning results indicated interactions 302 between antioxidants and emulsifier to a higher extent with Tween than Citrem, thus, resulting in a 303 higher concentration of antioxidant at the interface with Tween than Citrem as emulsifier. In 304 addition, the results indicated that there was a tendency to a lower concentration of caffeic acid and 305 methyl caffeate in the aqueous phase of the non-stripped Tween 80 emulsion, i.e. when endogenous 306 tocopherol was present. However, the differences were not significant (Table 2).

\section{Efficacy of caffeic acid and caffeates in emulsions}

The antioxidant efficacy of caffeic acid and caffeates were evaluated in the three different emulsions shown in Table 1 and in a modified CAT assay (modification: non-stripped tung oil

311 applied) to investigate the effect of the presence of endogenous tocopherols in this assay. The 312 efficacy of caffeic acid and caffeates was evaluated from the measured lipid oxidation during 313 storage. Lipid oxidation was followed by measuring PV (primary oxidation product) and volatiles 314 (secondary oxidation products). Volatile oxidation products that increased during storage were 315 identified and quantified. The quantified volatiles were markers of lipid oxidation products, most of 316 them originated from oxidation of n-3 PUFAs.

317 Citrem stabilized emulsions with endogenous tocopherols present. The PV in this experiment 318 increased to 20-35 meq. peroxides / $\mathrm{kg}$ oil depending on the antioxidant treatment during the 15 
days of storage (data not shown). The concentration of volatiles after 15 days of storage dependended upon the specific volatile quantified and the antioxidant applied (1-penten-3-one 20$60 \mathrm{ng} / \mathrm{g}$ emulsion, 1-penten-3-ol 100-300 ng / g emulsion, hexanal 70-150 ng / g emulsion, 4heptenal 10-25 ng / g emulsion, 2,4-heptadienal 4000-6000 ng / g emulsion and nonanal 30-70 ng / g emulsion). 
343 Tween stabilized emulsions with endogenous tocopherols present. In the Tween stabilized 344 emulsion with endogenous tocopherols present, the PV increased to 5-30 meq. peroxides / $\mathrm{kg}$ oil 345 depending on the antioxidant treatment during the 6 days of storage. The concentration of volatiles 346 after 6 days of storage dependent upon the specific volatile quantified and the antioxidant applied 347 (1-penten-3-one 2-10 ng / g emulsion, 1-penten-3-ol 5-30 ng / g emulsion and 2,4-heptadienal 50$348 \quad 350 \mathrm{ng} / \mathrm{g}$ emulsion).

349 A PCA of the PVs, $\alpha$-tocopherol and volatile compounds (1-penten-3-one, 1-penten-3-ol and 2,4350 heptadienal) measured during storage explained $82 \%$ of the variation in the obtained results by the 351 first two PCs (Figure 2). Similar to the other PCA model on Citrem stabilized emulsions, the 3 other 352 tocopherol homologues ( $\beta$-, $\delta$-, and $\gamma$-tocopherols) were excluded from the PCA model due to no or 353 minor changes during storage in the different emulsions. The PV and all the volatiles were located 354 to the left in the plot of the correlation loadings (Figure 2A). Tocopherols were located opposite to 355 the PV and volatiles. From the correlation loadings plot it is clear that the first PC describe lipid 356 oxidation with tocopherol in the right side and volatiles in the left side, thus, increased lipid 357 oxidation moving from the right to the left in the plot. Scores plot (Figure 2B) reveals differences in 358 efficacy between caffeic acid and caffeates in the tween stabilized emulsions. The control emulsion 359 was located to the left side of the PC 2 axis in the scores plot and all emulsions with antioxidant 360 added were located to the right of the PC 2 axis except emulsion with butyl caffeate added (Figure 361 2B). In connection with correlation loadings plot, this indicates that all the emulsions with 362 antioxidant added acted as antioxidant in the tween stabilized emulsions. As described above, butyl 363 caffeate was located opposite to the PC 2 axis compared to the other emulsions with antioxidant 364 added, this is explained by the higher amount of 2,4-heptadienal at day 6 in this emulsion (Figure 365 2). Butyl caffeate worked as antioxidant for all other oxidation parameters measured; however, it 366 was the least efficient due to higher amount of the measured oxidation parameters compared to the 
other antioxidant applied (Table 3). Moreover, the PCA model reveals that caffeic acid without esterification was more efficient in tween stabilized emulsions followed by methyl caffeate (short chain esterification) due to their location most far away from the oxidation parameters measured. Raw data supported the PCA model.

Tween80 stabilized emulsions without endogenous tocopherols present. In the Tween stabilized emulsion with endogenous tocopherols present, the PV increased from 8 to 14 meq. peroxides $/ \mathrm{kg}$ oil without antioxidant added during the 42 hours of storage; whereas, the PV increased from 2 to 8 and 1 to 4 meq. peroxides / $\mathrm{kg}$ oil with caffeic acid and eicosyl caffeates, respectively The other antioxidant treatments resulted in no increase in PV during storage. The concentration of volatiles after 42 hours of storage dependent upon the specific volatile quantified and the antioxidant applied (1-penten-3-one 0-3 ng / g emulsion, 1-penten-3-ol 0-9 ng / g emulsion and 2,4-heptadienal 2-90 ng / g emulsion), which was much lower than in the Citrem stabilized emulsions.

A PCA of the PVs and volatile compounds (1-penten-3-one, 1-penten-3-ol and 2,4-heptadienal) measured during storage explained $83 \%$ of the variation in the obtained results by the first to principal components (PCs), Figure 3. All the volatiles were located in quadrant 1 (top-right part) and 4 (bottom-right part). The first PC clearly describes lipid oxidation (right side) versus no lipid oxidation (left side), whereas, PC 2 describes the development of lipid oxidation over time, with PV and volatiles in the beginning of the storage period located in the top of the plot and in the bottom of the plot after 42 days (Figure 3A). Comparing Figure 3A with 3B, the scores plot, it is observed that control emulsion and emulsion with caffeic acid is located in the same side as the oxidation parameters measured. Thus, caffeic acid is acting as a prooxidant in Tween stabilized emulsions without endogenous tocopherol present. Esterification of caffeic acid in Tween stabilized emulsion without tocopherols improved its antioxidative properties, since all caffeates evaluated were acting as antioxidants. However, it seems like caffeic acid esterified with C20 was slightly less efficient 
391 than the other esters evaluated (Table 3). Raw data supports the observation from the PCA model 392 (data not shown).

393

394

395

396

397

398

399

400

401

402

403

404

405

406

407

408

409

410

411 The assay was slightly modified to investigate the efficacy of caffeic acid and caffeates in the

412 presence of endogenous tocopherols. The results are shown in Figure 4 together with results

413 obtained earlier with an unmodified CAT assay [8]. When tocopherol was present the antioxidative

Comparison of the influence of emulsifier type - Citrem versus Tween80 stabilized emulsions. Table 3 shows calculated inhibition percentages for caffeic acid and caffeates. It is clear that the emulsifier impacted the efficacy of the antioxidants added. In Citrem stabilized emulsions, caffeic acid and methyl caffeate were the only ones acting as antioxidants. Their antioxidative effect in this model emulsion was weak to intermediate and they even promoted the formation of certain volatiles. In contrast, caffeic acid and all the evaluated caffeates in Tween stabilized emulsions acted as antioxidants. Caffeic acid was the strongest antioxidant followed by methyl caffeate.

Comparison of the influence of the presence of endogenous tocopherols. Calculated inhibition percentages for selected oxidation variables measured in Tween stabilized emulsions with and without endogenous tocopherols are presented in Table 3. A clear difference in the efficacy of antioxidants was that caffeic acid acted as a strong antioxidant when tocopherols were present, whereas, it acted as intermediate to strong prooxidant without tocopherols in this model emulsion. In addition, the caffeates acted as stronger antioxidants without tocopherols compared to the same model emulsion with tocopherols present (Table 3). Caffeates with chain lengths between $\mathrm{C} 1$ and C18 were all strong antioxidants, whereas the antioxidant efficacy was decreased when the chain length was increased to $\mathrm{C} 20$.

Antioxidant efficacy in a modified CAT assay. The CAT assay is an assay developed to measure the efficacy of antioxidants in a micro emulsion system without endogenous tocopherols present. 
414 efficacies of caffeic acid, methyl-, butyl-, octyl- and dodecyl caffeates were not significantly 415 different. This finding is different from earlier results obtained without the presence of tocopherols, 416 where octyl- and dodecyl caffeates exerted a significantly higher efficacy than caffeic acid and the 417 other caffeates evaluated. Furthermore, the efficacy of octyl- and dodecyl caffeates without 418 tocopherols present was also significantly higher than when tocopherols were present. 
419

420

421 of emulsifier and the presence of endogenous tocopherols. Earlier measurements of the radical

422 scavenging effect (DPPH assay) of caffeic acid and caffeates showed no differences between the

423 caffeic acid and the different saturated unbranched alkyl esters [8]. In spite of that the antioxidative

424 effect of caffeic acid and caffeates was different in the different emulsion systems. However, the

425

426

427

428

429

430

431

432

433

434

435

436

437

438

439

440

441

442

\section{Discussion}

The results showed - as hypothesized - that partitioning of the antioxidants was affected by the type activity can differ due to the emulsion composition.

Impact of emulsifier on the efficacy of caffeic acid and caffeates. In the present study, caffeic acid and caffeates were more efficient antioxidants in Tween stabilized emulsions than in Citrem stabilized emulsions. This partly supports earlier findings with the same emulsifiers and caffeic acid in $10 \% \mathrm{O} / \mathrm{W}$ emulsions, where caffeic acid in Citrem stabilized emulsions promoted the formation of volatiles and no effect on lipid oxidation or slightly antioxidative effect of caffeic acid was observed in Tween stabilized emulsions [23]. Besides different oil concentration in the previous and current studies, antioxidant concentration (5.5 fold lower in this study) and oil / emulsifier ratio were also different, which can have an influence on the differences observed in these studies. Independently of emulsifier type, caffeic acid was performing better than the caffeates. Contrary, the effect of the caffeates was affected by the emulsifier applied. To our knowledge only few studies related to the antioxidative effect of caffeic acid and caffeates in emulsions have been published $[9,24,25]$. These studies did not compare the effect of the emulsifier type applied. However, one of them compared the effect of caffeates in mayonnaise and milk, where not only the emulsifier type is different but the entire emulsion system. The efficiency of the caffeates was affected by the type of emulsion system [9]. Results obtained in the present study also demonstrated that changing the emulsifier affected the antioxidative effect and rank order of caffeates. Experiments performed with gallic acid and ethyl gallate have also shown that changing emulsifier 
443 affects the partitioning of the antioxidants and the resulting antioxidant activity measured in 444 emulsion systems [26]. The different emulsifier evaluated was SDS, CTAB, Brij58 and PHLC, and their partitioning study revealed increased solubility effect of the emulsifiers in the following order: PHLC $<$ SDS $<$ Brij58 $<$ CTAB. The antioxidant activity of gallic acid and ethyl gallate based on the formation of hydroperoxides and hexanal increased in the following order: CTAB (no activity 448 measured $)<$ Brij58 $<$ PHLC $<$ SDS. Gallic acid only showed antioxidant activity with PHLC 449 stabilized emulsions. This was a reverse order compared to the partitioning measured. Hence, it was suggested that the increased partitioning into the emulsifier layer and lipid counteract the hydrogendonating ability, and lower the activity of the antioxidants [26]. Moreover, Pekkarinen et al. [27] evaluated antioxidative effect and partitioning of phenolics in different systems. Interaction between caffeic acid and Tween 20 differed from other phenolics such as vanillic acid, ferulic acid and 454 sinapic acid evaluated, since Tween 20 exhibited higher solubilisation capacity for caffeic acid than 455 for other phenolic acids. Additionally, Pekkarinen et al. [27] concluded that these antioxidant456 emulsifier interactions have a strong influence on the partitioning of antioxidants. The partitioning 457 result obtained in this study confirmed that Citrem and Tween as emulsifiers results in differences 458 in the partitioning of caffeic acid and caffeates. Less caffeic acid and caffeates were present in the 459 aqueous phase when Tween was applied. This clearly demonstrated stronger antioxidant-emulsifier 460 interaction with Tween compared to Citrem. Moreover, Citrem is an anionic emulsifier, thus, the 461 interface is negatively charged and will repel negatively charged antioxidants i.e. caffeic acid, 462 which could explain why caffeic acid interacted less with Citrem than with Tween. An explanation 463 for the stronger interactions with Tween may be the molecular structure of the emulsifiers, since 464 Tween is a larger and more bulky molecule than Citrem; however, this has to be further evaluated. 465 Furthermore, Schwarz et al. [19, 28, 29] evaluated partitioning of different antioxidants in dispersed 466 lipid systems with different emulsifiers. Significant differences were observed in partitioning of the 
467 antioxidants between phases, both as a function of $\mathrm{pH}$ and emulsifier type and concentration. It was 468 concluded from the results that determination of antioxidant partitioning may be an important tool 469 to select antioxidants structurally designed to localize at the surfaces [19], however, the partitioning 470 of the antioxidants cannot alone explain the measured antioxidant activity in emulsions [29].

471 Results from another study by Schwarz et al. [30] evaluating antioxidant activity of antioxidants

472 with different lipophilicity in bulk oil, $\mathrm{O} / \mathrm{W}$ and $\mathrm{W} / \mathrm{O}$ emulsions with different emulsifiers led to the 473 assumption that differences in antioxidant activity for the same emulsion type might be additionally 474 influenced by interaction with the emulsifier dominating the interfaces in the emulsion system [30]. 475 The obtained results for the two emulsifiers together with the partitioning study may also here lead 476 to the assumption that emulsifier-antioxidant interactions (e.g. hydrogen bonding) affected the 477 antioxidant activity of the caffeates. However, the type of interactions and the impact of 478 antioxidant-emulsifier interactions on the radical scavenging activity have to be studied in more 479 details to make further conclusions.

Impact of endogenous tocopherols on the efficacy of caffeic acid and caffeates. The presence of

481 endogenous tocopherol not only changed the antioxidant activity of caffeic acid and caffeates in 482 both the storage experiment and in the CAT assay, but also their partitioning in the emulsion 483 system. A tendency to less caffeic acid and caffeates ( $\mathrm{C} 1, \mathrm{C} 4$ and $\mathrm{C} 12)$ present in the aqueous phase 484 with endogenous tocopherol in the emulsion system was observed. This may indicate some 485 interactions between tocopherol and caffeic acid / caffeates both for the antioxidative effect and 486 localization in the emulsion system. The use of a combination of antioxidants to produce synergistic 487 interaction has been reported earlier e.g. tocopherol regeneration by ascorbic acid, polyphenols and 488 flavonoids [31-34]. Panya et al. [34] carried out the only study investigating interactions between 489 tocopherol and a phenol (rosmarinic acid) and its alkyl esters (rosmarinates, C4, C12 and C20) in 490 Tween20 stabilized emulsions. Rosmarinic acid exhibited strongest synergistic interaction with 
491 tocopherol, and $\mathrm{C} 4$ and $\mathrm{C} 12$ esters exhibited small synergistic interaction. An antagonistic 492 interaction was observed with C20 ester and tocopherol. Thus, the more hydrophilic rosmarinic acid 493 exhibited more interactions with the tocopheryl radical than the esters. In the present study, the 494 emulsion with the more hydrophilic caffeic acid exhibited better oxidative stability than the 495 emulsions with the esters (more hydrophobic antioxidants) when tocopherol was present as also 496 observed with rosmarinic acid and rosmarinates. Actually, caffeic acid turned from being 497 prooxidative without tocopherol present to being the most efficient antioxidant with endogenous 498 tocopherol present in Tween stabilized emulsion. In emulsions, the majority of the emulsifier is 499 accumulated at the oil-water interface. However, a part of the emulsifier is not associated with the 500 oil-water interface if the emulsifier concentration is above the CMC (critical micellar concentration, 501 CMC Tween80 13-15 mg/L, Sigma) and will form micelles in the aqueous phase. In this study, the 502 concentration of Tween was much higher than CMC $(10 \mathrm{~g} / \mathrm{L})$. The decreased antioxidant efficiency 503 of the different caffeates compared with caffeic acid is suggested to be due to the solubilisation of 504 caffeates in Tween micelles, thus, localized away from the interface unable to inhibit lipid 505 oxidation. Although, micelles are not isolated structures, they compromise structures that are in 506 dynamic equilibrium with other structures in the emulsion system. This means that components can 507 be exchanged between the different structures i.e. between micelles and emulsion droplets [35, 36]. 508 Thus, more studies are needed to further elucidate the differences observed in partitioning and 509 antioxidant effect in the presence of endogenous tocopherols.

510 Additionally, it is assumed that caffeic acid is located in close proximity to the interface where it 511 regenerates tocopherol at the interface in spite of the repelling effect of two negatively charged 512 compounds (Citrem and caffeic acid). The proposed partial location of tocopherol at the interface is 513 supported by Jacobsen et al. [37] who reported that ca. $6 \%$ of the alpha-tocopherol present in 514 mayonnaise was located at the interface whereas the remaining tocopherol was located in the oil 
515 phase. Therefore, lipophilization is not needed to improve the oxidative stability of these emulsions, 516 due to tocopherols location at the interface. Antioxidant hypotheses and efficacies of caffeic acid 517 and caffeates. The polar paradox was not confirmed in this study since caffeic acid was more 518 efficient as antioxidant than caffeates in Citrem stabilized emulsions when endogenous tocopherol 519 was present. In Tween stabilized emulsions with endogenous tocopherol caffeic acid followed by 520 methyl caffeate were the most efficient antioxidants, whereas, caffeic acid acted as a prooxidant 521 when tocopherol was not present. No cut-off effect was observed for the lipophilized caffeic acid, 522 since the most efficient antioxidant in both Citrem and Tween stabilized emulsions when tocopherol 523 was present was C0 (caffeic acid). A similar finding was observed for the CAT assay with 524 endogenous tocopherol present. Without endogenous tocopherol present, the caffeates were most 525 efficient antioxidants in Tween stabilized emulsions, a cut-off effect was found at C16. However, 526 the efficiency of C20 was still an intermediate to strong antioxidant. The CAT assay showed a cut527 off effect at around $\mathrm{C} 8$ and $\mathrm{C} 12$ with no endogenous tocopherol present. The partitioning 528 experiment clearly showed an effect of the chain length, with less antioxidant present in the aqueous 529 phase with increasing antioxidant lipophilicity. Pekkarinen et al. [27] observed that the proportion 530 of antioxidant solubilized in the lipid phase and particularly in the interface did not necessarily 531 reflect the efficiency of the antioxidant. It was assumed from their evaluation of antioxidant activity 532 and partitioning that specific interactions of the antioxidant with other compounds e.g. emulsifiers, 533 and intermolecular hydrogen bonds may play an important role in reducing antioxidant activity. 534 Furthermore, antioxidant-emulsifier interaction has a strong influence on partitioning of the 535 antioxidant. It is suggested that the caffeates interacts with the emulsifier in form of micelles when 536 endogenous tocopherols are present, resulting in reduced or no antioxidant activity. When 537 endogenous tocopherols are not present the caffeates is more likely to be solubilized at the interface 
538 instead of the tocopherols. However, this has to be evaluated more in depth to conclude further on 539 these differences in partitioning and antioxidant activity with the impact of endogenous tocopherols.

540 Conclusions. Partitioning and antioxidant activity of caffeic acid and caffeates were influenced 541 both by the emulsifier type and the presence of endogenous tocopherols. Thus, this study clearly 542 demonstrated different emulsifier-antioxidant and antioxidant-antioxidant interactions that affected 543 the efficacy of the evaluated caffeic acid and caffeates as antioxidant in emulsions. The hypotheses 544 about antioxidant in emulsions are based on simple emulsions systems without the presence of 545 tocopherols. However, the impact of the presence of tocopherols on the efficacy of other 546 antioxidants is important since most food systems contain tocopherol. 


\section{Acknowledgements}

548 We thank Thi Thu Trang Vu for skillful work in the laboratory, Maritex Norway (subsidiary of 549 TINE BA, Norway) and Danisco Ingredients (Dupont, Brabrand, Denmark) for providing the oils 550 and Citrem, respectively.

551 The study is a part of the project entitled "Phenolipids as antioxidants in omega-3 model and real

552 food systems - Effect of alkyl chain length and concentration" with project no 10-093655 financed

553 by the Danish Research Council, Technology and Production.

554 The authors declare no conflicts of interest. 


\section{Reference list}

556 1. McClements, D. J.; Decker, E. A. Lipid oxidation in oil-in-water emulsions: impact of molecular 557 environment on chemical reactions in heterogeneous food systems. J. Food Sci., 2000, 65, 1270$558 \quad 1282$.

559 2. Decker, E. A. Strategies for manipulating the prooxidative / antioxidative balance of foods to 560 maximize oxidative stability. Trends in Food Sci. Technol., 1998, 9, 241-248.

561 3. Porter, W. L. Paradoxical behavior of antioxidants in food and biological systems. Toxicol. Ind. 562 Health, 1993, 9, 93-122.

563 4. Laguerre, M.; Giraldo, L. J. L.; Lecomte, J.; Figueroa-Espinoza, M. C.; Barea, B.; Weiss, J.; 564 Decker, E. A.; Villeneuve, P. Chain Length Affects Antioxidant Properties of Chlorogenate Esters 565 in Emulsion: The Cutoff Theory Behind the Polar Paradox. J. Agric. Food Chem., 2009, 57, $11335-$ 56611342.

567 5. Frankel, E. N.; Huang, S.-W.; Kanner, J.; German, B. Interfacial phenomena in the evaluation of 568 antioxidants: bulk oils vs emulsions. J. Agric. Food Chem., 1994, 42, 1054-1059.

569 6. Laguerre, M.; Giraldo, L. J. L.; Lecomte, J.; Figueroa-Espinoza, M.-C.; Baréa, B.; Weiss, J.; 570 Decker, E. A.; Villeneuve, P. Relationship between hydrophobicity and antioxidant ability of 571 "phenolipids" in emulsion: a parabolic effect of the chain length of rosmarinate esters. J. Agric. 572 Food Chem., 2010, 58, 2869-2876.

573 7. Laguerre, M.; Sørensen, A.-D. M.; Bayrasy, C.; Lecomte, J.; Jacobsen, C.; Decker, E. A.; 574 Villeneuve, P. Role of hydrophobicity on antioxidant activity in lipid dispersions from the polar 575 paradox to the cut-off theory. In: Lipid oxidation: challenges in food systems. Ed. Logan, A.; 576 Nienaber, U.; Pan, X. AOCS Press, Urbana, IL. 2013 (p. 261-296) ISBN 9780983079163. 
577 8. Sørensen, A.-D. M.; Durand, E.; Laguerre, M.; Bayrasy, C.; Lecomte, J.; Villeneuve, P.;

578 Jacobsen, C. Antioxidant properties and efficacies of synthesized alkyl caffeates, ferulates and

579 coumarates. J. Agric. Food Chem., 2014, 62, 12553-12562.

580 9. Alemán, M.; Bou, R.; Guardiola, F.; Durand, E.; Villeneuve, P.; Jacobsen, C.; Sørensen, A.-D.

581 M. Antioxidative effect of lipophilized caffeic acid in fish oil enriched mayonnaise and milk. Food

582 Chem., 2015, 167, 236-244.

583 10. Panya, A.; Laguerre, M.; Bayrasy, C.; Lecomte, J.; Villeneuve, P.; McClements, D.J.; Decker,

584 E. A. An investigation of the versatile antioxidant mechanisms of action of rosmarinate alkyl esters

585 in oil-in-water emulsions. J. Agric. Food Chem., 2012, 60, 2692-2700.

586 11. Lee, J. H.; Panya, A.; Laguerre, M.; Bayrasy, C.; Lecomte, J.; Villeneuve, P.; Decker, E. A.

587 Comparison of the antioxidant capacities of rosmarinate alkyl esters in riboflavin photosensitized

588 oil-in-water emulsion. J. Am. Oil Chem. Soc., 2013, 90, 225-232.

589 12. Barden, L.; Barouh, N.; Villeneuve, P.; Decker, E. A. Impact of hydrophobicity on antioxidant

590 efficacy in low-moisture food. J. Agric. Food Chem., 2015, 63, 5821-5827.

591 13. AOCS Official Method Ce 8-89. Determination of Tocopherols and Tocotrienols in Vegetable

592 Oils and Fats by HPLC. Champaign, IL, USA, 1997.

593 14. Sørensen, A.-D. M.; Nielsen, N. S.; Yang, Z.; Xu, X.; Jacobsen, C. The effect of lipohilization 594 of dihydrocaffeic acid on its antioxidative properties in fish-oil-enriched emulsion. Eur. J. Lipid Sci.

595 Technol., 2012, 114, 134-145.

596 15. Rawle, A. Basic principles of particle size analysis. Malvern Instruments Ltd., 1996. 
597 16. Bligh, E. G.; Dyer, W. J. A rapid method of total lipid extraction and purification. Can. J.

598 Biochem. Physiol., 1959, 37, 911-917.

599 17. Iverson, S. J.; Lang, S. L. C.; Cooper, M. H. Comparison of the bligh and dyer and folch

600 methods for total lipid determination in broad range of marine tissue. Lipids, 2001, 36, 1283-1287.

601 18. Shantha, N. C.; Decker, E. A. Rapid, sensitive, iron-based spectrophotometric methods for 602 determination of peroxide values of food lipids. J. AOAC Int., 1994, 77, 421-424.

603 19. Schwarz, K.; Frankel, E. N.; German, J. B. Partition behaviour of antioxidative phenolic 604 compounds in heterophasic systems. Fett-Lipid 1996, 98, 115-121.

605 20. Sørensen, A.-D. M.; Nielsen, N. S.; Decker, E. A.; Let, M. B.; Xu, X.; Jacobsen, C. The efficacy 606 of compounds with different polarities as antioxidant in emulsions with omega-3 lipids. J. Am. Oil 607 Chem. Soc., 2011, 88, 489-502.

608 21. Laguerre, M.; Lopez-Giraldo, L. J.; Lecomte, J.; Barea, B.; Cambon, E.; Tchobo, P. F.; Barouh, 609 N.; Villeneuve, P. Conjugated autoxidizable triene (CAT) assay: A novel spectrophotometric 610 method for determination of antioxidant capacity using triacylglycerol as ultraviolet probe. Anal. 611 Biochem., 2008, 380, 282-290.

612 22. Jacobsen, C.; Let, M. B.; Nielsen, N. S.; Meyer, A. S.; Antioxidant strategies for preventing 613 oxidative flavor deterioration of food enriched with n-3 polyunsaturated lipids: a comparative 614 evaluation. Trends Food Sci. Technol., 2008, 19, 76-93.

615 23. Sørensen, A.-D. M.; Haarh, A.-M.; Becker, E. M.; Skibsted, L. H.; Bergenståhl, B.; Nilsson, L.; 616 Jacobsen, C. Interactions between iron, phenolic compounds, emulsifiers, and pH in omega-3617 enriched oil-in-water emulsions. J. Agric. Food Chem., 2008, 56, 1740-1750. 


\begin{abstract}
618 24. Costa, M.; Losada-Barreiro, S.; Paiva-Martins, F.; Bravo-Díaz, C.; Romsted, L. S. a direct
619 correlation between the antioxidant efficiencies of caffeic acid and its alkyl esters and their

620 concentrations in the interfacial region of olive oil emulsions. The pseudophase model

621 interpretation of the “cut-off”effect. Food Chem., 2015, 175, 233-242.

622 25. Costa, M.; Losada-Barreiro, S.; Paiva-Martins, F.; Bravo-Díaz, C. Optimizing the efficiency of

623 antioxidants in emulsions by lipophilization: tuning interfacial concentrations. RSC Adv., 2016, 6,

$62491483-91493$.
\end{abstract}

625 26. Stöckman, H.; Schwarz, K.; Huynh-Ba, T. The influence of various emulsifiers on the

626 partitioning and antioxidant activity of hydroxybenzoic acids and their derivatives in oil-in-water

627 emulsions. J. Am. Oil Chem. Soc., 2000, 77, 535-542.

628 27. Pekkarinen, S.S.; Stöckman, H.; Schwarz, K.; Heinonen, I.M.; Hopia, A.I. Antioxidant activity

629 and partitioning of phenolic acids in bulk and emulsified methyl linoleate. J. Agric. Food Chem., $6301999,41,3036-3043$.

631 28. Heins, A.; Garamus, V. M.; Steffen, B.; Stöckmann, H.; Schwarz, K. Impact of phenolic

632 antioxidants on structural properties of micellar solutions. Food Biophys., 2006, 1, 189-201.

633 29. Oehlke, K.; Heins, A.; Stöckmann, H.; Schwarz, K. Impact of emulsifier microenvironments on 634 acid-base equilibrium and activity of antioxidants. Food Chem., 2010, 118, 48-55.

635 30. Schwarz, K.; Huang, S.-W.; German, B.; Tiersch, B.; Hartmann, J.; Frankel, E.N. Activities of 636 antioxidants are affected by colloidal properties of oil-in-water and water-in-oil emulsions and bulk 637 oils. J. Agric. Food Chem., 2000, 48, 4874-4882.

638 31. Medina, I.; Undeland, I.; Larsson, K.; Storrø, I.; Rustad, T.; Jacobsen, C. Activity of caffeic acid 639 in different fish lipid matrices: a review. Food chem., 2012, 131, 730-740. 
640 32. Laranjinha, J.; Vieiva, O.; Madeira, V.; Almeida, L. Two related phenolic antioxidants with 641 opposite effects on vitamin E content in low density lipoproteins oxidized by ferrylmyoglobin:

642 composition vs regeneration. Arch. Biochem. Biophys., 1995, 323, 373-381.

643 33. Iglesias, J.; Pazos, M.; Andersen, M. L.; Skibsted, L. H.; Medina, I. Caffeic acid as antioxidant 644 in fish muscle: mechanism of synergism with endogenous ascorbic acid and alpha-tocopherol. J. 645 Agric. Food Chem., 2009, 57, 675-681.

646 34. Panya, A.; Kittipongpittaya, K.; Laguerre, M.; Bayrasy, C.; Lecomte, J.; Villeneuve, P.; 647 McClements, D.J.; Decker, E.A. Interactions between $\alpha$-tocopherol and rosmarinic acid and its 648 alkyl esters in emulsions: synergistic, additive, or antagonistic effect? J. Agric. Food Chem., 2012, $64960,10320-10330$.

650 35. Skhiri, Y.; Gruner, P.; Semin, B.; Brosseau, Q.; Pekin, D.; Mazutis, L.; Goust, V.;

651 Kleinschmidt, F.; Harrak, A. E.; Hutchison, J. B.; Mayot, E.; Bartolo, J.-F.; Griffiths, A. D.; Taly, 652 V.; Baret, J.-C. Dynamics of molecular transport by surfactants in emulsions. Soft Matter, 2012, 8, $653 \quad 10618-10627$.

654 36. You, Y.; Bloomfield, A.; Liu, J.; Fu, L.; Herzon, S. B.; Yan, C. E. Real-time kinetics of 655 surfactant molecule transfer between emulsion particles probed by in situ second harmonic 656 generation spectroscopy. J. Am. Oil Chem. Soc., 2012, 134, 4264-4268.

657 37. Jacobsen, C.; Schwarz, K.; Stoeckmann, H.; Meyer, A.S.; Adler-Nissen, J. Partitioning of 658 selected antioxidants in mayonnaise. J. Agric. Food Chem., 1999, 47, 3601-3610. 


\section{$659 \quad$ Figure legends}

660 Figure 1 PCA build on results obtained from PV, volatiles (1-penten-3-one, 1-penten-3-ol, 4661 heptenal, 2,4-heptadienal, hexanal and nonanal) and $\alpha$-tocopherols measured on Citrem stabilized 662 emulsions during storage (15 days) using full cross validation. A) Correlation loadings and B) 663 Scores plot. Abbreviations for sample codes refer to Table 1.

664 Figure 2 PCA build on results obtained from PV, volatiles (1-penten-3-one, 1-penten-3-ol and 2,4665 heptadienal) and $\alpha$-tocopherols measured on Tween80 stabilized emulsions during storage (6 days) 666 using full cross validation. A) Correlation loadings and B) Scores plot. Abbreviations for sample 667 codes refer to Table 1.

668 Figure 3 PCA build on results obtained from PV and volatiles (1-penten-3-one, 1-penten-3-ol and 669 2,4-heptadienal) measured on Tween80 stabilized emulsions during storage (42 hours) using full 670 cross validation. A) Correlation loadings and B) Scores plot. Abbreviations for sample codes refer 671 to Table 1.

672 Figure 4 CAT Value of caffeic acid and caffeates (C1-C16) measured in the concentration range of $6730.5-2 \mu \mathrm{M}$. $\bullet$ CAT Values determined without endogenous tocopherols (normal condition for the 674 CAT assay, published in Sørensen et al. [8]) and o CAT Values determined with endogenous 675 tocopherols (modified CAT assay). 
677 Table 1 Experimental design of experiment 1 and 2.

\begin{tabular}{|c|c|c|c|c|}
\hline Experiment & Sample code & Emulsifier & Oil & Antioxidant \\
\hline & C_Con & Citrem & $\mathrm{FO}$ & No antioxidant \\
\hline & C_CA C0 & Citrem & FO & Caffeic acid \\
\hline $\mathrm{E}$ & C_CA C1 & Citrem & FO & Methyl caffeate \\
\hline $\mathrm{X}$ & $\mathrm{C}_{-} \mathrm{CAC} 4$ & Citrem & FO & Butyl caffeate \\
\hline $\mathrm{P}$ & C_CA C8 & Citrem & $\mathrm{FO}$ & Octyl caffeate \\
\hline \multirow{3}{*}{1} & C_CA C12 & Citrem & $\mathrm{FO}$ & Dodecyl caffeate \\
\hline & C_CA C16 & Citrem & FO & Hexadecyl caffeate \\
\hline & $\mathrm{C}_{-} \mathrm{CA} \mathrm{C} 20$ & Citrem & $\mathrm{FO}$ & Eicosyl caffeate \\
\hline & T_Con & Tween80 & $\mathrm{FO} / \mathrm{RO}$ & No antioxidant \\
\hline & T_CA C0 & Tween80 & $\mathrm{FO} / \mathrm{RO}$ & Caffeic acid \\
\hline & $\mathrm{T}_{-} \mathrm{CAC} 1$ & Tween80 & $\mathrm{FO} / \mathrm{RO}$ & Methyl caffeate \\
\hline & $\mathrm{T}_{-} \mathrm{CAC} 4$ & Tween80 & $\mathrm{FO} / \mathrm{RO}$ & Butyl caffeate \\
\hline $\mathrm{E}$ & $\mathrm{T}_{-} \mathrm{CAC} 8$ & Tween80 & $\mathrm{FO} / \mathrm{RO}$ & Octyl caffeate \\
\hline$X$ & T_CA C12 & Tween 80 & $\mathrm{FO} / \mathrm{RO}$ & Dodecyl caffeate \\
\hline $\mathrm{P}$ & T_CA C16 & Tween 80 & $\mathrm{FO} / \mathrm{RO}$ & Hexadecyl caffeate \\
\hline \multirow{8}{*}{2} & TS_Con & Tween 80 & $\mathrm{~S} \mathrm{FO} / \mathrm{RO}$ & No antioxidant \\
\hline & TS_CAC0 & Tween80 & $\mathrm{S} \mathrm{FO} / \mathrm{RO}$ & Caffeic acid \\
\hline & TS_CA C1 & Tween80 & $\mathrm{S} \mathrm{FO} / \mathrm{RO}$ & Methyl caffeate \\
\hline & TS_CA C4 & Tween80 & $\mathrm{S} \mathrm{FO} / \mathrm{RO}$ & Butyl caffeate \\
\hline & TS_CA C8 & Tween80 & $\mathrm{S} \mathrm{FO} / \mathrm{RO}$ & Octyl caffeate \\
\hline & TS_CA C12 & Tween80 & $\mathrm{S} \mathrm{FO} / \mathrm{RO}$ & Dodecyl caffeate \\
\hline & TS_CA C16 & Tween80 & $\mathrm{S} F O / \mathrm{RO}$ & Hexadecyl caffeate \\
\hline & TS_CA C20 & Tween80 & $\mathrm{S} \mathrm{FO} / \mathrm{RO}$ & Eicosyl caffeate \\
\hline
\end{tabular}

678 Abbreviations: FO Fish oil, FO/RO Fish oil and rapeseed oil (1:1, w/w) and S FO/RO Stripped fish

679 oil and rapeseed oil $(1: 1, \mathrm{w} / \mathrm{w})$

680 
681 Table 2 Concentration $[\mu \mathrm{M}]$ of caffeic acid and caffeates (Methyl, Butyl, Octyl and Dodecyl) measured in the aqueous phase of different systems: 95\% Buffer / 5\% Oil, 99\% Buffer / 1\% Emulsifier and Emulsion (5\% Oil, 1\% Emulsifier and 94\% Buffer). Citrem and Tween80

683 were applied as emulsifier. Both non-stripped and stripped FO/RO was evaluated with Tween80 as emulsifier.

\begin{tabular}{|c|c|c|c|c|c|c|c|c|}
\hline \multirow[b]{2}{*}{ Antioxidant } & \multicolumn{3}{|c|}{ Citrem and non-stripped oil } & \multicolumn{3}{|c|}{ Tween80 and non-stripped oil } & \multicolumn{2}{|c|}{ Tween80 and stripped oil } \\
\hline & Buffer / Oil & Buffer / Emulsifier & Emulsion & Buffer / Oil & $\begin{array}{c}\text { Buffer / } \\
\text { Emulsifier }\end{array}$ & Emulsion & Buffer / Oil & Emulsion \\
\hline$\overline{\mathrm{CA} \mathrm{CO}}$ & $101 \pm 3.1^{\mathrm{a}, \mathrm{b}, \mathrm{x}}$ & $91.5 \pm 3.6^{6, x}$ & $91.5 \pm 7.1^{\mathrm{b}, \mathrm{x}}$ & $93.7 \pm 11.4^{b, x}$ & $75.4 \pm 7.0^{\mathrm{c}, \mathrm{x}}$ & $74.0 \pm 4.3^{\mathrm{c}, \mathrm{x}}$ & $111 \pm 2.6^{\mathrm{a}, \mathrm{x}}$ & $78.4 \pm 10.1^{\mathrm{c}, \mathrm{x}}$ \\
\hline CA C1 & $86.2 \pm 0.9^{\mathrm{a}, \mathrm{y}}$ & $53.3 \pm 3.0^{\mathrm{b}, \mathrm{y}}$ & $43.8 \pm 8.2^{\mathrm{b}, \mathrm{y}}$ & $82.8 \pm 1.9^{\mathrm{a}, \mathrm{x}}$ & $15.4 \pm 3.3^{\mathrm{c}, \mathrm{y}}$ & $10.1 \pm 2.5^{\mathrm{c}, \mathrm{y}}$ & $91.1 \pm 2.6^{\mathrm{a}, \mathrm{y}}$ & $15.3 \pm 3.1^{\mathrm{c}, \mathrm{y}}$ \\
\hline CA C4 & $11.4 \pm 0.4^{\mathrm{a}, \mathrm{b}, \mathrm{z}}$ & $4.09 \pm 0.6^{\mathrm{a}, \mathrm{b}, \mathrm{c}, \mathrm{z}}$ & $3.39 \pm 0.5^{\mathrm{b}, \mathrm{c}, \mathrm{z}}$ & $11.3 \pm 0.7^{\mathrm{a}, \mathrm{b}, \mathrm{y}}$ & $2.10 \pm 0.3^{\mathrm{c}, \mathrm{z}}$ & $<$ detection & $13.0 \pm 1.1^{\mathrm{a}, \mathrm{z}}$ & $<$ detection \\
\hline CA C8 & $<$ detection & $<$ detection & $<$ detection & $<$ detection & $2.60 \pm 3.6^{\mathrm{z}}$ & $<$ detection & $<$ detection & $<$ detection \\
\hline CA C12 & $<$ detection & $<$ detection & $<$ detection & $2.00 \pm 0.6^{\mathrm{b}, \mathrm{z}}$ & $1.10 \pm 0.4^{\mathrm{z}}$ & $<$ detection & $9.70 \pm 4.2^{\mathrm{a}, \mathrm{z}}$ & $1.10 \pm 0.2^{\mathrm{b}, \mathrm{y}}$ \\
\hline
\end{tabular}

684 Different letters in superscript indicate significant differences. Significant differences within a row i.e. same antioxidant but different systems are denoted with a,b and

c, whereas significant differences within a column i.e. same system but different antioxidant are denoted with $\mathrm{x}, \mathrm{y}$ and $\mathrm{z}$. 
Table 3 Calculated inhibition percentages for selected lipid oxidation parameters (PV, 1-penten-3-ol, 1-penten-3-one and 2,4-

\begin{tabular}{|c|c|c|c|c|c|c|c|c|c|c|c|c|}
\hline \multirow{2}{*}{$\mathrm{AO}$} & \multicolumn{4}{|c|}{ Citrem (Day 6) } & \multicolumn{4}{|c|}{ Tween with tocopherols } & \multicolumn{4}{|c|}{ Tween without tocoherols } \\
\hline & PV & 1Pen3ol & 1Pen3one & 2,4HepA & PV & 1Pen3ol & 1Pen3one & 2,4HepA & PV & 1Pen3ol & 1Pen3one & 2,4HepA \\
\hline$\overline{\mathrm{CA}}$ & 50 & 39 & 3 & 44 & 79 & 83 & 86 & 86 & 34 & -63 & -55 & -48 \\
\hline $\mathrm{C} 1$ & 32 & 1 & -210 & -27 & 60 & 60 & 69 & 11 & 89 & 97 & 85 & 89 \\
\hline $\mathrm{C} 4$ & 21 & -119 & -502 & -143 & 47 & 61 & 33 & -10 & 89 & 99 & 83 & 93 \\
\hline $\mathrm{C} 8$ & -28 & -174 & -603 & -87 & 49 & 71 & 52 & -3 & 88 & 100 & 94 & 94 \\
\hline $\mathrm{C} 12$ & -53 & -130 & -515 & -13 & 43 & 62 & 55 & 2 & 91 & 102 & 97 & 95 \\
\hline $\mathrm{C} 16$ & -26 & -104 & -487 & -39 & 44 & 58 & 39 & 4 & 87 & 101 & 96 & 98 \\
\hline $\mathrm{C} 20$ & -31 & -43 & -340 & 8 & & & & & 66 & 59 & 44 & 45 \\
\hline \multirow{2}{*}{$\mathrm{AO}$} & \multicolumn{4}{|c|}{ Citrem (Day 15) } & & & & & & & & \\
\hline & PV & 1Pen3ol & & $2,4 \mathrm{HepA}$ & & & & & & & & \\
\hline$\overline{\mathrm{CA}}$ & 13 & -166 & 52 & 13 & & & & & & & & \\
\hline $\mathrm{C} 1$ & 31 & -123 & 65 & 18 & & & & & & & & \\
\hline $\mathrm{C} 4$ & 28 & -61 & -65 & -41 & & & & & & & & \\
\hline $\mathrm{C} 8$ & 54 & -47 & -51 & -17 & & & & & & & & \\
\hline $\mathrm{C} 12$ & 5 & -10 & -28 & 5 & & & & & & & & \\
\hline C16 & -7 & 3 & -21 & 5 & & & & & & & & \\
\hline $\mathrm{C} 20$ & -5 & 12 & -15 & 7 & & & & & & & & \\
\hline
\end{tabular}

689 Abbreviation: AO Antioxidant; PV Peroxide Value; 1Pen3ol 1-Penten-3-ol; 1Pen3one 1-Penten-3-one; 2,4HepA 2,4-HeptadienalA. The 2,4-heptadienal external

690 standard appears as two peaks in the chromotogramme, these peaks are termed A and B (here only 2,4HepA presented). 


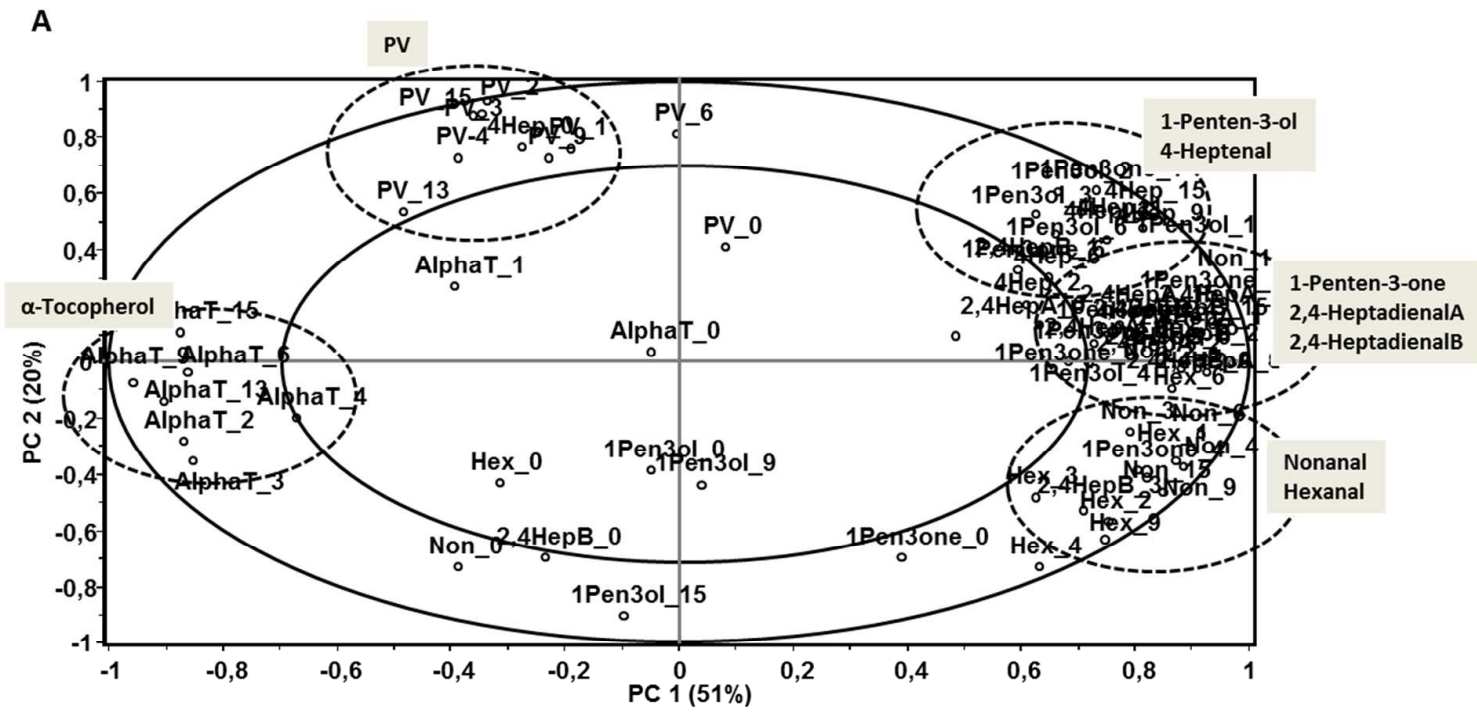

692

$$
\text { B }
$$

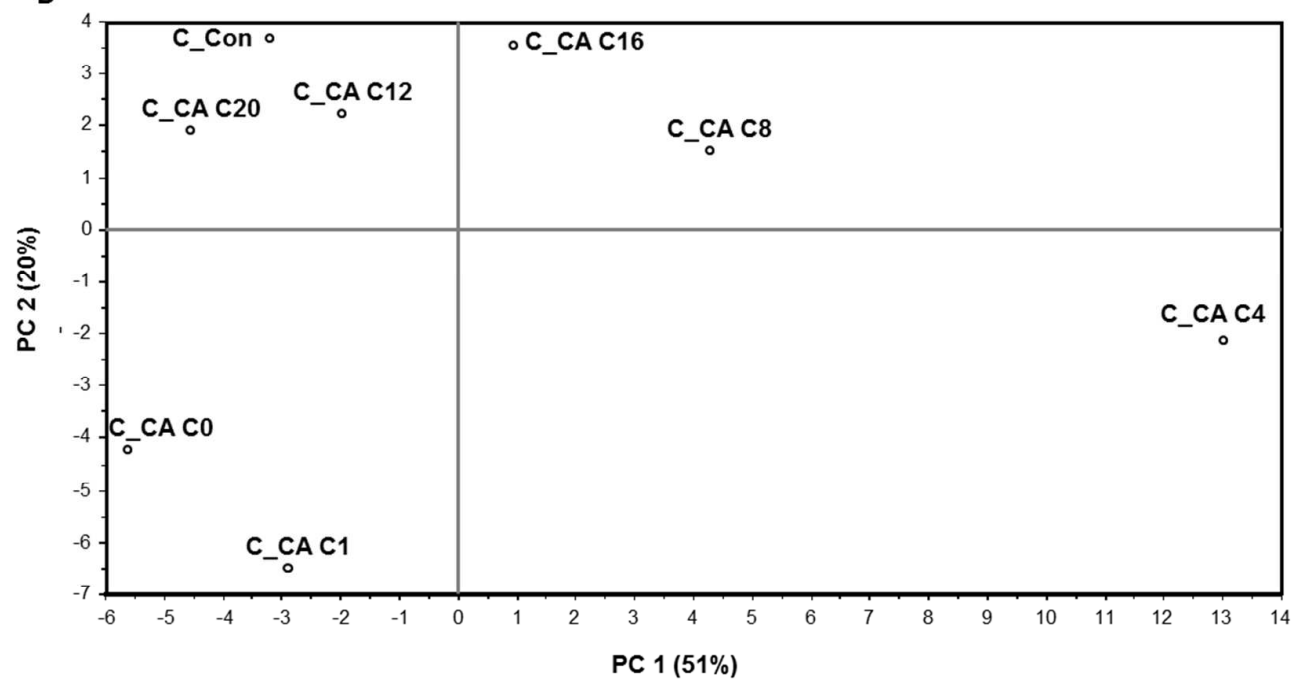

693

\section{Figure 1}

695 


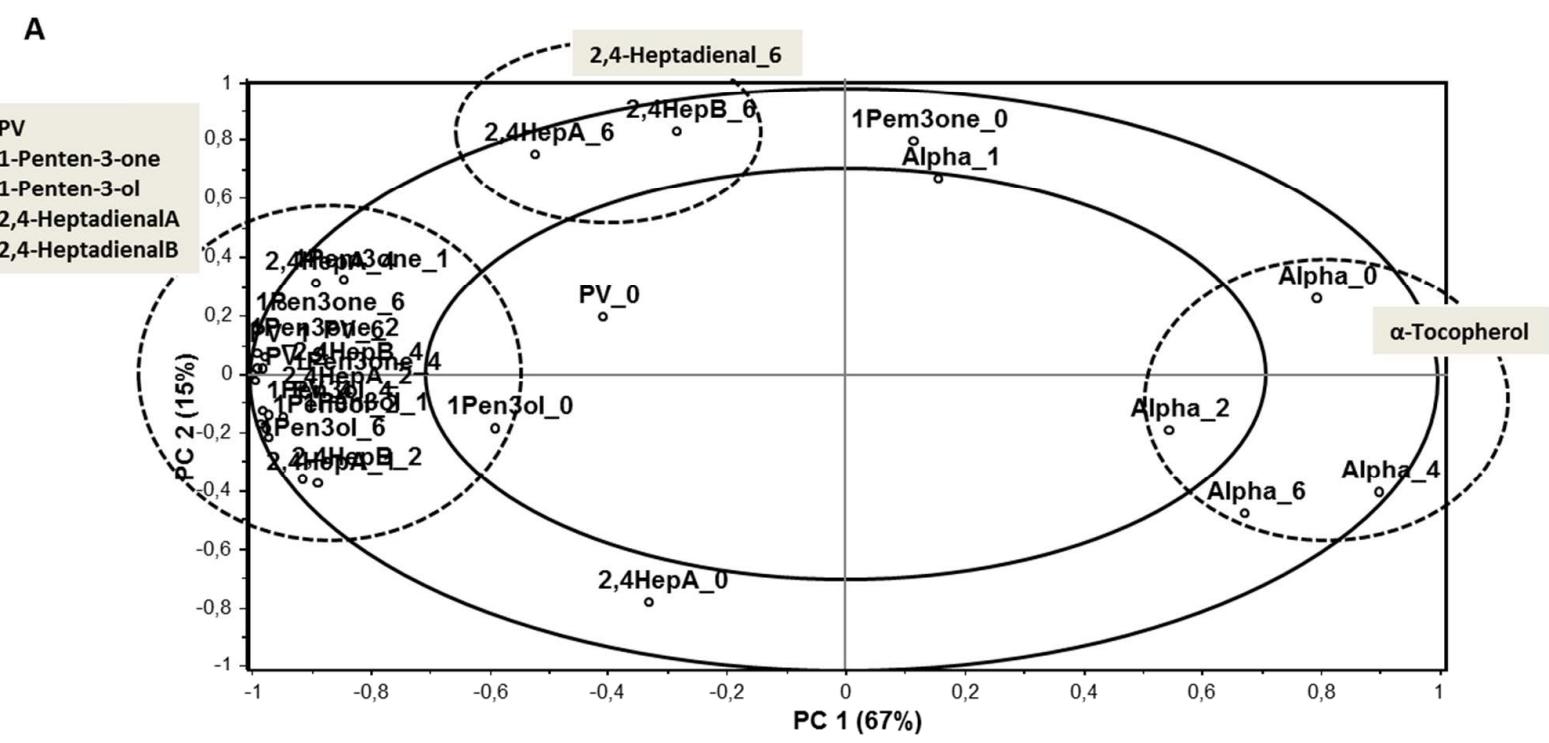

696

697

B

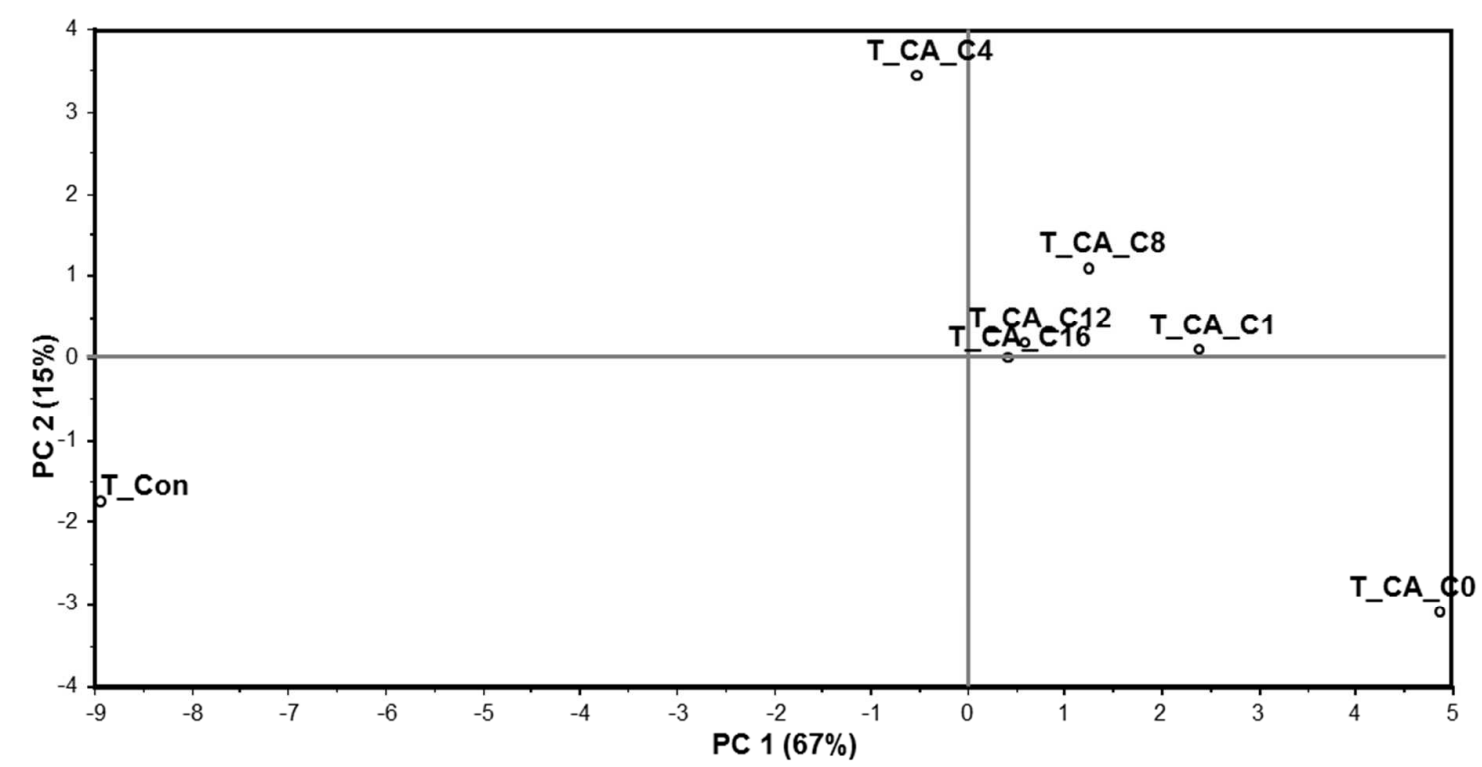

698

699 Figure 2

700

701 
A

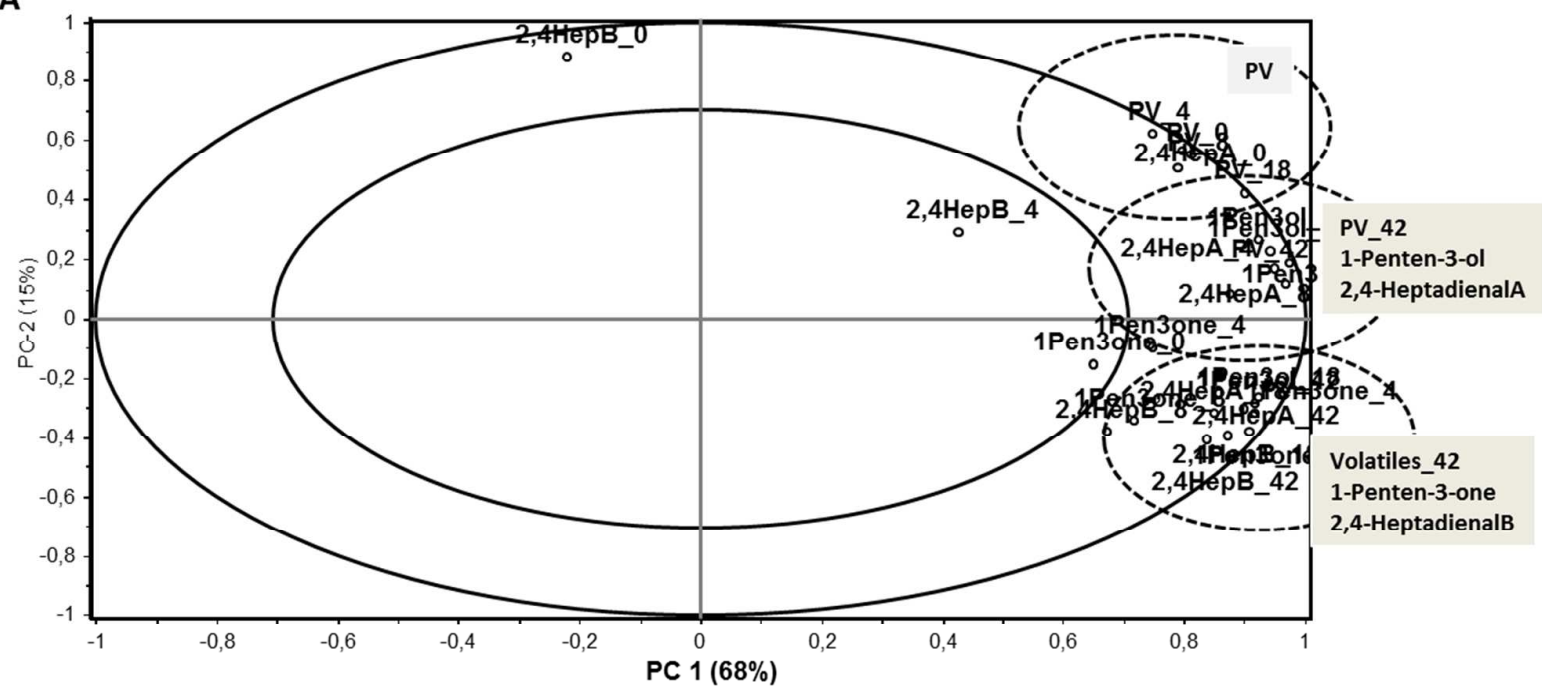

702

B

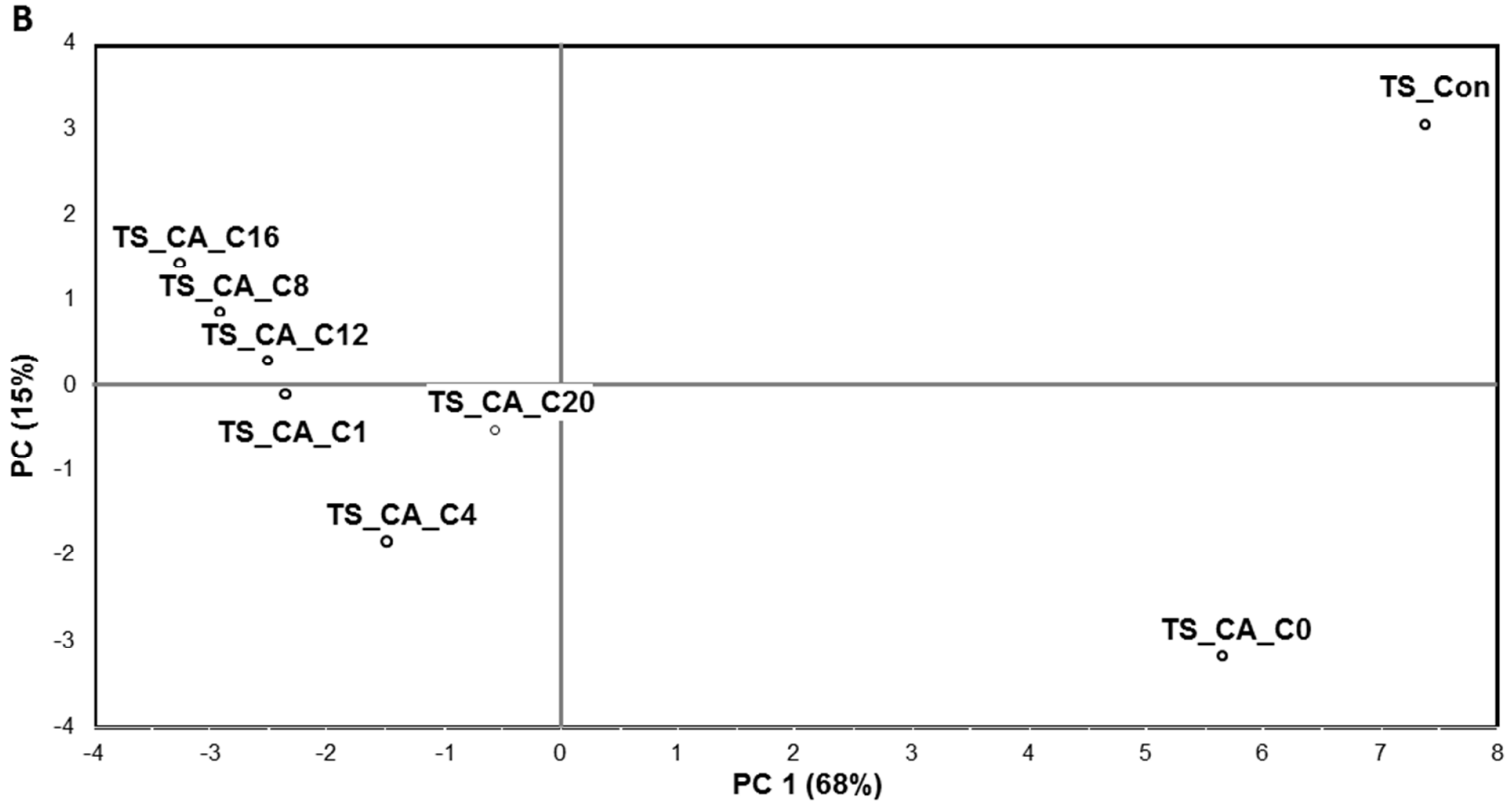

703

Figure 3 


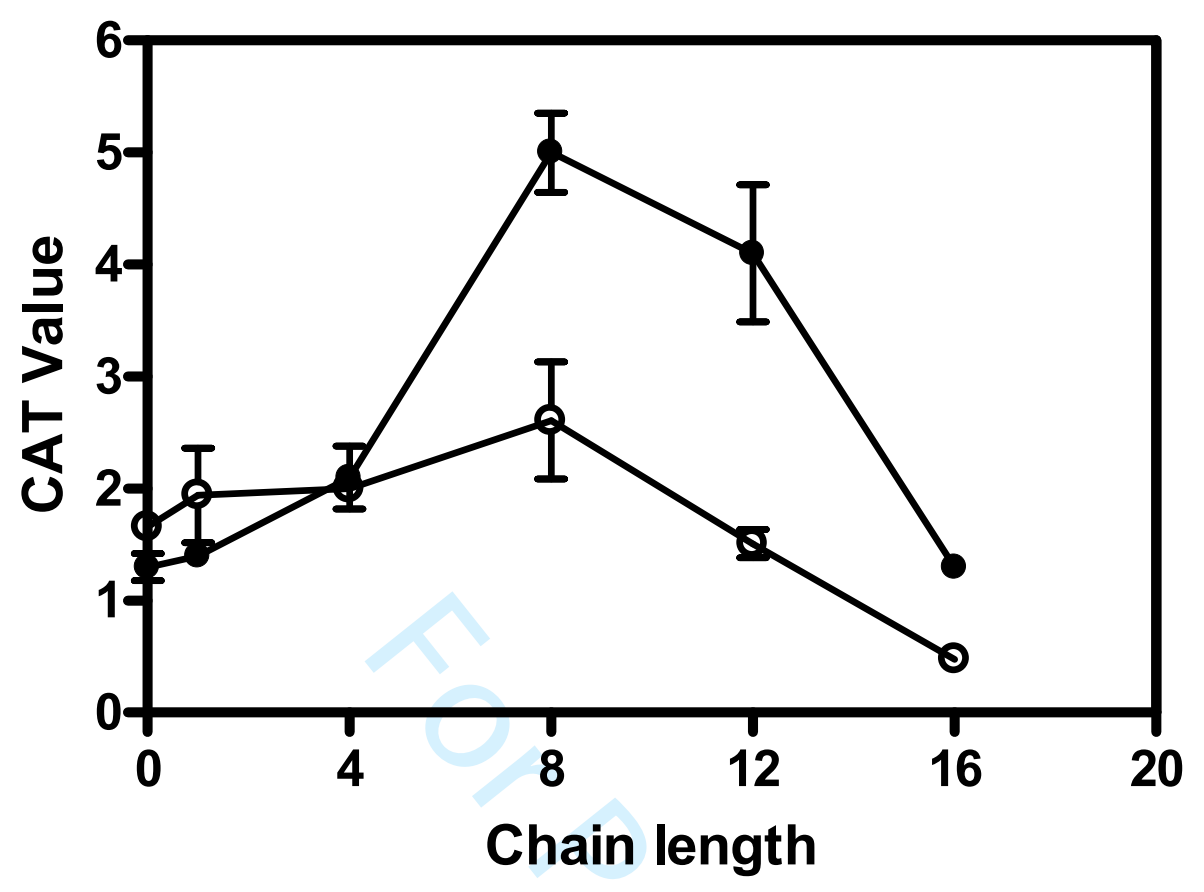

705

Figure 4

707

708

709

710

711 
1 Alkyl caffeates as antioxidants in $\mathrm{\theta} / \mathrm{w} \underline{\mathrm{O} / \mathrm{W}}$ emulsions: Impact of emulsifier type and endogenous 2 tocopherols

${ }^{1}$ Division of Food Technology, National Food Institute (DTU Food), Technical University of

Denmark, DK-2800 Kgs. Lyngby, Denmark

${ }^{2}$ CIRAD, UMR IATE, Montpellier, F-34398, France

*Corresponding author: adms@food.dtu.dk, Phone: +45 45252591

11

Running title: Oxidation is affected by emulsifier and antioxidant-Effect of emulsifier and antioxidants on lipid oxidation

Keywords: Caffeic acid; Lipid oxidation; Tween; Citrem; Phenolipids; Fish oil; Antioxidant interactions

Abbreviations: CA Caffeic acid; C1 Methyl caffeate; C4 Butyl caffeate; C8 Octyl caffeate; C12 Dodecyl caffeate; C16 Hexadecyl caffeate; C20 Eicosyl caffeate; CAT Conjugated autoxidizable triene; CCL Critical chain length; FO Fish oil; FO/RO Fish oil and rapeseed oil mixture (1:1); PC Principal component; PCA Principal component analysis; PV Peroxide value; THF Tetrahydrofuran 


\section{Abstract}

Antioxidant addition can be one strategy to limit lipid oxidation in emulsions. Research has proven that an important factor regarding the efficacy of antioxidants is their localization in the emulsion; however, other factors such as interactions with other components can also have an impact. Thus, the aim was to evaluate the impact of emulsifiers (Citrem and Tween 80 ) and presence of endogenous tocopherols on the efficacies of caffeic acid and caffeates (C1-C20) as antioxidants in emulsions. Lipid oxidation was evaluated during storage and partitioning of caffeic acid and caffeates was estimated by measuring their concentrations in the aqueous phase.

Partitioning of caffeic acid and caffeates was influenced by emulsifier type and presence of endogenous tocopherols. Caffeic acid was the most efficient antioxidant in Citrem and Tween stabilized emulsions in the presence of endogenous tocopherol. In contrast, for Tween stabilized emulsions, caffeic acid acted as a prooxidant and the evaluated caffeates acted as strong antioxidants in the absence of endogenous tocopherol. Thus, when endogenous tocopherol was present lipophilization of caffeic acid did not increase its efficacy as an antioxidant. It is suggested that the differences observed in antioxidant efficiency with different emulsifiers and with and without endogenous tocopherols is due to emulsifier-antioxidant interactions and antioxidantantioxidant interactions in the emulsions.

Practical application: Food emulsions contain endogenous tocopherols, thus, the impact of endogenous tocopherols on the efficacy of applied antioxidants is of interest to the industry. So far the hypotheses about antioxidant in emulsions are based on simple emulsions systems without the presence of tocopherols. The finding in this study revealed that both emulsifier type and the presence of endogenous tocopherol had an impact on the efficacy of caffeic acid and caffeates due to emulsifier-antioxidant and antioxidant-antioxidant interactions. This highlights the importance of 
843 evaluating the antioxidant in each emulsion system before selecting antioxidants for optimal 9 1044 protection against lipid oxidation.

11

1245

13

1446

15

16

17

18

19

20

21

22

23

24

25

26

27

28

29

30

31

32

33

34

35

36

37

38

39

40

41

42

43

44

45

46

47

48

49

50

51

52

53

54 
An o/w emulsion consists of three different phases. A dispersed phase (oil) is present as droplets in a continuous phase (water) and separated by an interfacial region. Type of emulsions can range from very simple, when prepared from only a few ingredients, to more complex, when prepared with many different ingredients e.g. food emulsions. Lipid oxidation can occur rapidly in emulsions due to their large interfacial area. The interface region facilitates interactions between the lipids and water-soluble prooxidants [1].

Different strategies can be applied to limit lipid oxidation and thereby improve shelf life of emulsions [2]. One of them is addition of antioxidants; however, selection of the right antioxidant or mixture of antioxidants is difficult, since their efficacies are affected by the composition of the emulsions e.g. their localization and interaction with other components. An enormous amount of studies have been reported in the literature on antioxidants and their efficacies in model emulsions and more complex food emulsions. So far, two hypotheses about antioxidant efficacies in emulsions, namely the polar paradox hypothesis [3] and the cut-off effect [4] have been reported. In brief, the polar paradox hypothesizes that apolar antioxidants are more efficient in $\mathrm{O} / \mathrm{W}$ emulsions than polar antioxidants [3] due to differences in the antioxidants' affinity towards the different phases [5]. The cut-off effect can be seen as an extension of the polar paradox hypothesis, in which an optimal degree of lipophilisation for optimal antioxidant activity is observed [4], which is called critical chain length (CCL). The cut-off effect was observed from results obtained with chlorogenic acid and rosmarinic acid and their unbranched saturated alkyl esters (chlorogenates and rosmarinates). The efficacy of antioxidant homologues was related to the partitioning of these antioxidants in an emulsion system [4, 6]. Based on these observations, it was assumed that when the lipophilized antioxidants had the CCL they were present in the highest concentration at the oilaqueous interface, where lipid oxidation is initiated. In addition, antioxidant homologues with chain 
71 length below and above CCL were driven away from the oil-aqueous interface [7]. These antioxidant hypotheses are based on extensive research in simplified emulsions prepared with stripped oils, whereas food emulsions contain e.g. endogenous tocopherols. Only few compounds with different degree of lipophilization have been evaluated in several different emulsion systems. Caffeic acid and unbranched saturated caffeates have been evaluated both in a model emulsion (CAT assay) system [8], milk and mayonnaise [9] and CCL seemed to be influenced by the system. In addition, rosmarinic acid has also been evaluated in different systems; model emulsions and low moisture food (crackers). In model emulsions, a parabolic relationship between antioxidant efficacy and hydrophobicity was observed, with the intermediate polarity $(8$ carbon -18 carbon chain length) giving optimum activity $[6,10,11]$. In crackers, a linear relationship between antioxidant activity and hydrophobicity was observed [12].

Due to formerly obtained results with caffeic acid and caffeates in model emulsion, milk and mayonnaise [8,9], it is hypothesized that emulsifier type and the presence of endogenous tocopherols can affect the partitioning of caffeic acid and caffeates and thereby change their efficacy in the emulsion systems. Hence, the aim of this study was to evaluate the impact of both emulsifier and presence of endogenous tocopherols on the efficacies of caffeic acid and caffeates (C1-C20). Two experiments were carried out as shown in Table 1. In the first experiment, the aim was to evaluate the effect of lipophilization of caffeic acid on its antioxidant efficacy in an $\theta / \mathrm{wO} / \mathrm{W}$ emulsion prepared with unstripped oil and Citrem as emulsifier. The aim of the second experiment was to investigate whether the presence of endogenous tocopherol affected the optimal chain length of lipophilized caffeic acid when Tween was used as an emulsifier. Moreover, comparison of results of the two experiments enabled an evaluation of whether the emulsifier type, Citrem vs Tween affected the CCL in emulsions prepared with unstripped oil. 
894 In both experiments, the partitioning of the antioxidant was estimated by measuring its 9

1095 concentration in the aqueous phase of the $\mathrm{\theta} / \mathrm{O} / \mathrm{W}$ emulsion, a buffer/oil system and a 1196 buffer/emulsifier system to evaluate if emulsifier type and the presence of endogenous tocopherols, 1397 affected the partitioning of caffeic acid and caffeates in the emulsion system. 
Material and Methods

The oil used in this study was fish oil (FO) and a mixture between fish and rapeseed oil (FO/RO, 1:1). The two types of oil were supplied by Maritex A/S (TINE BA, Sortland, Norway). The quality of the FO was as follows: 0.3 meq. peroxides / $\mathrm{kg}$ oil, $250 \mathrm{mg} \alpha$-tocopherol / $\mathrm{kg}, 98 \mathrm{mg} \gamma$ tocopherol / $\mathrm{kg}$ and $48 \mathrm{mg} \delta$-tocopherol $/ \mathrm{kg}$. Fatty acid composition of the fish oil was as follows: $14: 0,3.5 \% ; 16: 0,9.9 \% ; 16: 1 n-7,8.8 \% ; 18: 0,2.0 \% ; 18: 1 n-9,16.3 \% ; 18: 1 n-7,4.9 \% ; 18: 2 n-6,1,8 \%$, $18: 3 n-3,2.6 \%, \quad 18: 4 n-3,2.6 \%, 20: 1 n-7,12.6 \% ; 20: 5 n-3$ (EPA), 9.16\%; 22:1n-9, 5.8\%, 22:5n-3, $1.1 \%$ and $22: 6 n-3$ (DHA) $11.1 \%$. The total percentages of $n-3$ and n-6 PUFA in the FO were $24.0 \%$ and $1.8 \%$, respectively. The quality of the FO/RO was as follows: 0.3 meq. peroxides / $\mathrm{kg}$ oil, 230 $\mathrm{mg} \alpha$-tocopherol / kg, $31 \mathrm{mg} \beta$-tocopherol / kg, $151 \mathrm{mg} \gamma$-tocopherol / $\mathrm{kg}$ and $6 \mathrm{mg} \delta$-tocopherol / $\mathrm{kg}$. The fatty acid composition of the FO/RO was as follows: 14:0, 1.7\%; 16:0, 7.1\%; 16:1 (n-7), $4.6 \% ; 18: 0,1.9 \% ; 18: 1$ (n-9), 38.3\%; 18:1 (n-7), 3.3\%; 18:2 (n-6), 10.5\%, 18:3 (n-3), 4.8\%; 18:4 (n-3), 1.2\%; 20:1, 6.9\%; 20:5 (n-3, EPA), 4.7\%; 22:1 (n-11), 3.0\% and 22:6 (n-3, DHA), 5.9\%. The total content of n-3 and n-6 PUFA in the FO/RO were $17.8 \%$ and $10.9 \%$, respectively.

The emulsifiers applied, Tween 80 and Citrem LR 10 Extra (citric acid ester of mono- and diglyceride) without antioxidants were supplied by Sigma Aldrich (Steinheim, Germany) and Dupont (Danisco A/S, Grindsted, Denmark), respectively.

Alkyl caffeates were synthesized in an acid catalyzed reaction with caffeic acid and fatty alcohols with alcohol in excess as reaction medium or THF (tetrahydrofuran) as reaction medium. For further details refer to Sørensen et al. [8].

Tung oil (872 g/mol), Brij 35 (a nonionic polyoxyethylene surfactant, estimated Mw $1198 \mathrm{~g} / \mathrm{mol}$ ), AAPH (2,2' azobis-2 methyl-propanimidamide, dihydrochloride), phosphate buffer solution (PBS, pH 7.2), alumina, BHT (butylated hydroxytoluene), AAPH (2,2'-Azobis(2-methylpropionamidine) 
dihydrochloride) and trolox were purchased from Sigma-Aldrich (Steinheim, Germany). Synperonic was purchased from CRODA (East Yorkshire, UK). All solvents used were of HPLC grade and purchased from Lab-Scan (Dublin, Ireland). The external standards used for quantification of secondary oxidation products were from Sigma Aldrich (Steinheim, Germany).

\section{Experimental design}

For specific details on emulsifier, oil and antioxidants applied refer to Table 1. Experiment 1 was designed to evaluate the effect caffeic acid and different alkyl caffeates as antioxidant in Citrem stabilized emulsions in the presence of endogenous tocopherols due to the natural content of tocopherols in oil. In this experiment fish oil was used, and a storage period of 15 days selected based on previous storage experiment with fish oil.

Experiment 2 was designed to evaluate the influence of endogenous tocopherols on the efficacy of caffeic acid and caffeates in Tween stabilized emulsions. Tween was selected as emulsifier, since Citrem contains tocopherols. Emulsions were prepared with and without endogenous tocopherols. Thus, the oil without endogenous tocopherols was stripped (removal of tocopherols). Stripping of fish oil will increase oxidation rates tremendously. To slow down oxidation rate, a mixture of fish and rapeseed oil was therefore used instead in this experiment. The storage time was selected based on preliminary laboratory trials (data not shown).

Based on experiment 1 and 2 with endogenous tocopherols, the impact of emulsifier type is evaluated despite different oil type and length of storage time.

\section{Removal of tocopherols from oils}


FO/RO was stripped from tocopherols using an alumina packed glass column using hexane. For further details refer to Sørensen et al [8]. The stripped oil was bottled, flushed with nitrogen and stored at $-80^{\circ} \mathrm{C}$ until use for production of emulsions. Furthermore, the absence of tocopherols in the oil was checked by HPLC according to the AOCS method [13]. After oil stripping (removal of tocopherols) the PV was 0.5 meq. peroxides / $\mathrm{kg}$ oil and tocopherols were not detected.

\section{Production of $\theta^{\prime} / \underline{\mathrm{O} / \mathrm{W}}$ emulsions}

Both in experiment 1 and 2, the emulsion compositions were 5\% oil, $1 \%$ emulsifier and 94\% 10 $\mathrm{mM}$ sodium acetate - imidazole buffer $(\mathrm{pH} 7)$. Antioxidants were diluted in methanol and added in concentrations of $100 \mu \mathrm{M}$. For the control emulsions (without antioxidant added), methanol was added in same amount as used for the methanolic antioxidant solutions added to the other emulsions. The short to medium chain phenolipids $(\mathrm{C} 0-\mathrm{C} 12)$ were added to the buffer (Citrem emulsions) and buffer-emulsifier mixture (Tween emulsions), whereas the long chain phenolipids (C16 - C20) were added to the oil-emulsifier mixture (Citrem emulsions) and oil (Tween emulsions) before the pre-homogenisation step.

Preparation of emulsions for storage experiment 1 were produced with pre-emulsification $(2 \mathrm{~min}$, Ultra-Turrax, Janke \& Kunkel IKA-Labortechnik, Staufen, Germany) followed by homogenization on a two-valve table homogenizer at a pressure of 800 bar (GEA Niro Soavi Spa, Parma, Italy). For further details refer to Sørensen et al. [14]. Production of emulsions for storage experiment 2 were pre-emulsified as in experiment 1 , but homogenized on a microfluidizer $(9 \mathrm{~K}$, Microfluidics, Newton, MA, USA). Changes in the production between experiment 1 and 2 (homogenizer vs. microfluidizer) wasere done in order to operate with smaller emulsion volumes and; thereby reduce the amount of phenolipids. Moreover, it became possible to, and the ability to-cool the emulsion 
during production which was an advantage due to the fact that when-the oil was stripped from tocopherols and would therefore be highly susceptible to oxidation during homogenization.

After production emulsions $(100 \mathrm{~g})$ were stored in $100 \mathrm{~mL}$ blue cap bottles at $20^{\circ} \mathrm{C} \pm 2^{\circ} \mathrm{C}$. Samples, one bottle pr. code, were taken at specific time points and divided into brown glass bottles, flushed with nitrogen and stored at $-40^{\circ} \mathrm{C}$ until analyses, except for samples used to determine droplet size, these samples were measured at the sampling day without pre-freezing.

\section{Droplet size measurements}

Droplet size of the oil droplets in the $\mathrm{\theta} / \mathrm{w} \mathrm{O} / \mathrm{W}$ emulsion was determined by laser diffraction (Mastersizer2000, Malvern Instruments Ltd., Worcestershire, UK). Few droplets of the different emulsions were suspended directly in recirculating water (2800 rpm, obscuration 12-14\%). Water $\left(\mathrm{RI}_{\text {water }}=1.330\right)$ and sunflower oil $\left(\mathrm{RI}_{\mathrm{oil}}=1.469\right)$ were used in this measurements as dispersant and particle, respectively. Each sample was measured in triplicate $(n=3)$ and results are reported as surface mean diameter, $\mathrm{D}_{3,2}[15]$.

\section{Lipid extractions from $\theta / w \underline{\mathrm{O} / \mathrm{W}}$ emulsions}

Peroxide value (PV) and tocopherol analyses are performed on lipid extracts. Thus, the lipids were extracted from the emulsions prior to these analyses according to the method described by Bligh and Dyer [16] using a reduced amount of solvent [17]. For each sample code two lipid extractions were performed $(\mathrm{n}=2)$. 
Lipid extracts were evaporated under nitrogen, re-dissolved in heptane and analyzed by HPLC (Agilent 1100 Series, Agilent Technology, Palo Alto, CA, USA) according to the AOCS Official Method Ce 8-89 [13]. A silica column (Waters (Dublin, Ireland), 150mm, 4.6mm, $3 \mu \mathrm{m}$ silica film) was used for separation of the tocopherol homologues. This analysis was performed in duplicate on each lipid extract and results reported as $\mu \mathrm{g}$ tocopherol / $\mathrm{g}$ emulsion.

\section{Primary oxidation products: Peroxide value (PV)}

PVs in the lipid extracts were determined by colorimetric method based on formation of an ironthiocyanate complex. The colored complex was measured on a spectrophotometer at $500 \mathrm{~nm}$ (Shimadzu UV1800, Shimadzu Scientific Instruments, Columbia, MD, USA) [18]. The analysis was performed in duplicate and reported as meq peroxides / $\mathrm{kg}$ oil.

\section{Secondary oxidation products: Volatiles}

Volatiles were released from the $\mathrm{e} / \mathrm{wO} / \mathrm{W}$ emulsion using dynamic headspace $\left(45^{\circ} \mathrm{C}\right.$ for $30 \mathrm{~min},{ }^{*}$ nitrogen flow of $150 \mathrm{~mL} / \mathrm{min}$ ). Volatiles were then collected and trapped on Tenax GR packed tubes. To avoid foam and thus water on the tubes, $4 \mathrm{~mL}$ of antifoam (Synperonic, conc. $8 \mathrm{~g} / \mathrm{L}$ water) was added to each sample prior to the collection. Trapped volatiles were desorbed using an automatic thermal desorber (ATD-400, Perkin Elmer, Waltham, MA, USA). The transfer line of the ATD was connected to a gas chromatograph (Agilent 5890, Palo Alto, CA, USA) with a mass selective detector (HP 5972). Volatiles were separated on a DB1701 column (30m x ID 0.25mm x $1 \mu \mathrm{m}$ film thickness, J\&W Scientific, Folsom, CA, USA). The initial temperature of the oven was $45^{\circ} \mathrm{C}$, which was kept for 5 minutes and then gradually increased as follows: $45-55^{\circ} \mathrm{C} 1.5^{\circ} \mathrm{C} / \mathrm{min}$, 
$55-90^{\circ} \mathrm{C} 2.5^{\circ} \mathrm{C} / \mathrm{min}, 90-220^{\circ} \mathrm{C} 12^{\circ} \mathrm{C} / \mathrm{min}$ and kept at $220^{\circ} \mathrm{C}$ for 4 minutes. Calibration curves prepared from external standards was used for quantification of different volatiles. In experiment 1 (Table 1), different concentrations of external standard solutions were prepared and $1 \mu \mathrm{L}$ was placed in Tenax tubes and analyzed. In experiment 2 (Table 1), different concentrations of external standard solutions were prepared and added to fresh emulsion without antioxidant. Volatiles were collected in the same way as for samples. The 2,4-heptadienal external standard appears as two peaks in the chromotogramme, these peaks are termed A and B. Each sample code was analyzed in triplicate $(\mathrm{n}=3)$ and results reported as ng volatile / $\mathrm{g}$ emulsion.

\section{Partitioning}

Partitioning of caffeic acid and the different alkyl caffeates in buffer/oil, emulsifier/buffer and in ब/wO/W emulsion was measured according to the method described by Schwarz et al. [19] with modifications as described elsewhere. With this method it is assumed that the partitioning of the antioxidants, equilibra reached, is n'ot disrupted by centrifugation. For further details refer to Sørensen et al. [20].

In short, the concentration of antioxidants was measured in the separated aqueous phase of 3 different systems: buffer / oil (FO or FO/RO), buffer / emulsifier (Citrem or Tween80) and 5\% $\mathrm{\theta} / \mathrm{WO} / \mathrm{W}$ emulsions. Antioxidants were dissolved in methanol and added in a concentration of 100 $\mu \mathrm{M}$. Separation of the aqueous phase was carried out $24 \mathrm{~h}$ after production of the different systems.

\section{Determination of concentration of added antioxidant}


Caffeic acid and alkyl caffeates in the aqueous phases were analyzed by HPLC (Agilent 1100

Series, Agilent Technology, CA, USA) with a C18 Thermo Hypersil ${ }^{\circ}$ ODS $(250 \times 4.6$ mm, $5 \mu)$ column and using a gradient elution at a flow rate of $1 \mathrm{~mL} / \mathrm{min}$. Solvent A was $3 \mathrm{mM}$ phosphoric acid and solvent B was methanol. Gradient condition: $0-30$ min 0-100\% B, 30-40 min 100\% B and 40-45 min 100-0\% B. Injection volume was $20 \mu \mathrm{L}$. Caffeic acid and different alkyl caffeates were quantified by calibration curves of these compounds dissolved in methanol.

\section{Conjugated autoxidizable triene (CAT) Assay}

Stock solutions of the different compounds: caffeic acid, caffeates and trolox were prepared in methanol. The CAT assay was performed as described in Sørensen et al. [8] with a modification. This assay was performed with non-stripped tung oil, hence, the assay contained tocopherol and the evaluated antioxidant in a mixture. Each microplate well contained a microemulsion with the following composition: $115 \mu \mathrm{M}$ tung oil, $17 \mu \mathrm{M}$ Brij 35, $1 \mathrm{mM}$ AAPH and antioxidant (caffeic acid, caffeates or Trolox) in various concentrations. The progress of lipid oxidation was followed by measuring the decrease in absorbance at $273 \mathrm{~nm}$. Each antioxidant concentration was measured in triplicate on the plate and via independent measurements (two different microplates), $n=6$. Results were expressed as CAT value (mean $\pm \mathrm{SD}$ ). This method was developed by Laguerre et al. [21]. For further details about the calculations refer to Laguerre et al. [4, 21].

\section{Data treatment}

Statistics. The results obtained were analyzed using one- and two-way ANOVA (GraphPad Prism, Version 4.01, GraphPad Software Inc). Bonferroni multiple comparison post-test was used to determine significant differences between samples or storage times. The significance level applied 
Multivariate data analysis, Principal Component Analysis (PCA). Inhibition percentages calculated from results obtained from PV, volatiles and tocopherol analysis were subjected to PCA using Unscrambler version X10.3 (Camo, Oslo, Norway). The PCA model was built on inhibition percentages calculated on the average of the measured data. Full cross validation was used to validate the model. A PCA allows detection of similarities and dissimilarities between the different samples in a score plot, whereas correlations between the measured variables are visualized in a loadings plot. Connecting these plots in this case shows the degree of oxidation between the different samples. 


\section{Partitioning of caffeic acid and caffeates}

The concentration of caffeic acid (CA) and caffeates (CA C1 - CA C12) were determined in the aqueous phase of three different systems to mimic the applied emulsion systems. Hence, the two different emulsifiers and non-stripped and stripped oil was applied. The results obtained are presented in Table 2. In general, the partitioning of caffeic acid and caffeates in the aqueous phase decreased with increased degree of lipophilization until chain length $\mathrm{C} 4$ after which the caffeates in most cases could not be detected in the aqueous phase. In the case of the buffer/Tween system 


\section{Efficacy of caffeic acid and caffeates in emulsions}

The antioxidant efficacy of caffeic acid and caffeates were evaluated in the three different emulsions shown in Table 1 and in a modified CAT assay (modification: non-stripped tung oil applied) to investigate the effect of the presence of endogenous tocopherols in this assay. The efficacy of caffeic acid and caffeates was evaluated from the measured lipid oxidation during storage. Lipid oxidation was followed by measuring PV (primary oxidation product) and volatiles (secondary oxidation products). Volatile oxidation products that increased during storage were identified and quantified. The quantified volatiles were markers of lipid oxidation products, most of them originated from oxidation of n-3 PUFAs. 
Citrem stabilized emulsions with endogenous tocopherols present. The PV in this experiment $10^{320}$ 11321 $12^{321}$ $13_{322}$ 14 15323 16 17324 18 19325 20 increased to 20-35 meq. peroxidaes / $\mathrm{kg}$ oil depending on the antioxidant treatment during the 15 days of storage (data not shown). The concentration of volatiles after 15 days of storage dependended upon the specific volatile quantified and the antioxidant applied (1-penten-3-one 20$60 \mathrm{ng} / \mathrm{g}$ emulsion, 1-penten-3-ol 100-300 ng / g emulsion, hexanal 70-150 ng / g emulsion, 4heptenal 10-25 ng / g emulsion, 2,4-heptadienal 4000-6000 ng / g emulsion and nonanal 30-70 ng / g emulsion).

A PCA of the PVs, $\alpha$-tocopherol and volatile compounds measured during storage explained $71 \%$ of the variation in the obtained results by the first two principal components (PCs), Figure 1 . The 3 other tocopherols ( $\beta$-, $\delta$-, and $\gamma$-tocopherols) were excluded from the PCA model due to no or minor changes during storage in the different emulsions. Figure 1A shows the correlation loadings i.e. graphical mapping of the measured variables. Generally, all the volatiles were located in quadrant 1 (top-right part) and 4 (bottom-right part), PVs in quadrant 2 (top-left part) closer to PC 2 and $\alpha$ tocopherols in quadrant 2 and 3 (bottom-left part) a long PC 1. Thus, the first PC clearly described lipid oxidation with tocopherol in the left side and volatiles in the right side. Comparing Figure $1 \mathrm{~A}$ with $1 \mathrm{~B}$, the scores, reveals the differences in efficacy between caffeic acid and caffeates. Butyl caffeate was prooxidative due to its location in the 4th quadrant i.e. high concentration of many of the volatile compounds. Octyl caffeate also acted as a prooxidant, however, not as strong as butyl caffeate. Furthermore, hexadecyl caffeate's location in first quadrant, but close to PC 2, indicates a slight prooxidative effect. Dodecyl and eicosyl caffeates seemed to have no effect due to their proximity to the control emulsion in the 2 nd quadrant. The location of caffeic acid in the 3 quadrant of the scores plot away from the volatile compounds ( 1 and 4 quadrant) indicates that this antioxidant was the most efficient followed by methyl caffeate. However, caffeic acid and methyl caffeate were not strong antioxidants, since some of the measured volatiles were present in higher 
concentration in these emulsions than in the control emulsion (Table 3, Inhibition percentages, Raw data not shown).

Tween stabilized emulsions with endogenous tocopherols present. In the Tween stabilized emulsion with endogenous tocopherols present, the PV increased to 5-30 meq. peroxides / $\mathrm{kg}$ oil depending on the antioxidant treatment during the 6 days of storage. The concentration of volatiles after 6 days of storage dependent upon the specific volatile quantified and the antioxidant applied (1-penten-3-one 2-10 ng / g emulsion, 1-penten-3-ol 5-30 ng / g emulsion and 2,4-heptadienal 50$350 \mathrm{ng} / \mathrm{g}$ emulsion).

A PCA of the PVs, $\alpha$-tocopherol and volatile compounds (1-penten-3-one, 1-penten-3-ol and 2,4heptadienal) measured during storage explained $82 \%$ of the variation in the obtained results by the first two PCs (Figure 2). Similar to the other PCA model on Citrem stabilized emulsions, the 3 other tocopherol homologues ( $\beta$-, $\delta$-, and $\gamma$-tocopherols) were excluded from the PCA model due to no or minor changes during storage in the different emulsions. The PV and all the volatiles were located to the left in the plot of the correlation loadings (Figure 2A). Tocopherols were located opposite to the PV and volatiles. From the correlation loadings plot it is clear that the first PC describe lipid oxidation with tocopherol in the right side and volatiles in the left side, thus, increased lipid oxidation moving from the right to the left in the plot. Scores plot (Figure 2B) reveals differences in efficacy between caffeic acid and caffeates in the tween stabilized emulsions. The control emulsion was located to the left side of the PC 2 axis in the scores plot and all emulsions with antioxidant added were located to the right of the PC 2 axis except emulsion with butyl caffeate added (Figure 2B). In connection with correlation loadings plot, this indicates that all the emulsions with antioxidant added acted as antioxidant in the tween stabilized emulsions. As described above, butyl caffeate was located opposite to the PC 2 axis compared to the other emulsions with antioxidant added, this is explained by the higher amount of 2,4-heptadienal at day 6 in this emulsion (Figure 
2). Butyl caffeate worked as antioxidant for all other oxidation parameters measured; however, it was the least efficient due to higher amount of the measured oxidation parameters compared to the other antioxidant applied (Table 3). Moreover, the PCA model reveals that caffeic acid without esterification was more efficient in tween stabilized emulsions followed by methyl caffeate (short chain esterification) due to their location most far away from the oxidation parameters measured. Raw data supported the PCA model.

Tween 80 stabilized emulsions without endogenous tocopherols present. In the Tween stabilized emulsion with endogenous tocopherols present, the PV increased to-from $\theta \underline{8}-\underline{\text { to }} 14$ meq. peroxidzes / $\mathrm{kg}$ oil without antioxidant addeddepending on the antioxidant treatment during the 42 hours of storage; whereas, the PV increased from 2-to 8 and 1- to 4 meq. Pperoxides / kg oil with caffeic acid and eicosyl caffeates, respectively- The other antioxidant treatments resulted in no increase in PV during storage. - The concentration of volatiles after 42 hours of storage dependent upon the specific volatile quantified and the antioxidant applied (1-penten-3-one 0-3 ng / g emulsion, 1penten-3-ol 0-9 ng / g emulsion and 2,4-heptadienal 2-90 ng / g emulsion), which was much lower than in the Citrem stabilized emulsions.

A PCA of the PVs and volatile compounds (1-penten-3-one, 1-penten-3-ol, 4-heptenal and 2,4heptadienal) measured during storage explained $83 \%$ of the variation in the obtained results by the first to principal components (PCs), Figure 3. All the volatiles were located in quadrant 1 (top-right part) and 4 (bottom-right part). The first PC clearly describes lipid oxidation (right side) versus no lipid oxidation (left side), whereas, PC 2 describes the development of lipid oxidation over time, with PV and volatiles in the beginning of the storage period located in the top of the plot and in the bottom of the plot after 42 dayshere increasing oxidation has decreasing PC 2 values (Figure $3 \mathrm{~A}$ ). Comparing Figure $3 \mathrm{~A}$ with $3 \mathrm{~B}$, the scores plot, it is observed that control emulsion and emulsion with caffeic acid is located in the same side as the oxidation parameters measured. Thus, caffeic 
acid is acting as a prooxidant in Tween stabilized emulsions without endogenous tocopherol present. Esterification of caffeic acid in Tween stabilized emulsion without tocopherols improved its antioxidative properties, since all caffeates evaluated were acting as antioxidants. However, it seems like caffeic acid esterified with C20 was slightly less efficient than the other esters evaluated (Table 3). Raw data supports the observation from the PCA model (data not shown).

\section{Comparison of the influence of emulsifier type - Citrem versus Tween $\underline{80}$ stabilized emulsions.}

Table 3 shows calculated inhibition percentages for caffeic acid and caffeates. It is clear that the emulsifier impacted the efficacy of the antioxidants added. In Citrem stabilized emulsions, caffeic acid and methyl caffeate were the only ones acting as antioxidants. Their antioxidative effect in this model emulsion was weak to intermediate and they even promoted the formation of certain volatiles. In contrast, caffeic acid and all the evaluated caffeates in Tween stabilized emulsions acted as antioxidants. Caffeic acid was the strongest antioxidant followed by methyl caffeate.

Comparison of the influence of the presence of endogenous tocopherols. Calculated inhibition percentages for selected oxidation variables measured in Tween stabilized emulsions with and without endogenous tocopherols are presented in Table 3. A clear difference in the efficacy of antioxidants was that caffeic acid acted as a strong antioxidant when tocopherols were present, whereas, it acted as intermediate to strong prooxidant without tocopherols in this model emulsion. In addition, the caffeates acted as stronger antioxidants without tocopherols compared to the same model emulsion with tocopherols present (Table 3). Caffeates with chain lengths between $\mathrm{C} 1$ and C18 were all strong antioxidants, whereas the antioxidant efficacy was decreased when the chain length was increased to C20.

Antioxidant efficacy in a modified CAT assay. The CAT assay is an assay developed to measure the efficacy of antioxidants in a micro emulsion system without endogenous tocopherols present. 
The assay was slightly modified to investigate the efficacy of caffeic acid and caffeates in the presence of endogenous tocopherols. The results are shown in Figure 4 together with results obtained earlier with an unmodified CAT assay [8]. When tocopherol was present the antioxidative efficacies of caffeic acid, methyl-, butyl-, octyl- and dodecyl caffeates were not significantly different. This finding is different from earlier results obtained without the presence of tocopherols, where octyl- and dodecyl caffeates exerted a significantly higher efficacy than caffeic acid and the other caffeates evaluated. Furthermore, the efficacy of octyl- and dodecyl caffeates without tocopherols present was also significantly higher than when tocopherols were present. 


\section{Discussion}

The results showed - as hypothesized - that partitioning of the antioxidants was affected by the type of emulsifier and the presence of endogenous tocopherols. Earlier measurements of the radical scavenging effect (DPPH assay) of caffeic acid and caffeates showed no differences between the caffeic acid and the different saturated unbranched alkyl esters [8]. In spite of that the antioxidative effect of caffeic acid and caffeates was different in the different emulsion systems. However, the activity can differ due to the emulsion composition.

Impact of emulsifier on the efficacy of caffeic acid and caffeates. In the present study, caffeic acid and caffeates were more efficient antioxidants in Tween stabilized emulsions than in Citrem stabilized emulsions. This partly supports earlier findings with the same emulsifiers and caffeic acid in $10 \% \mathrm{e} / \mathrm{W} / \mathrm{W}$ emulsions, where caffeic acid in Citrem stabilized emulsions promoted the formation of volatiles and no effect on lipid oxidation or slightly antioxidative effect of caffeic acid was observed in Tween stabilized emulsions [23]. Besides different oil concentration in the previous and current studies, antioxidant concentration (5.5 fold higher $\underline{\text { lower in the present this }}$ study) and oil / emulsifier ratio were also different, which can have an influence on the differences observed in these studies. Independently of emulsifier type, caffeic acid was performing better than the caffeates. Contrary, the effect of the caffeates was affected by the emulsifier applied. To our knowledge Oonly few studies related to the antioxidative effect of caffeic acid and, caffeates in emulsions have been published $[9,24,25]$. These studies did not compare the effect of the emulsifier type applied. However, one of them compared the effect of caffeates in mayonnaise and milk, where not only the emulsifier type is different but the entire emulsion system. The efficiency of the caffeates was affected by the type of emulsion system [9]. Results obtained in the present study also demonstrated that changing the emulsifier affected the antioxidative effect and rank order of caffeates. Experiments performed with gallic acid and ethyl gallate have also shown that 
changing emulsifier affects the partitioning of the antioxidants and the resulting antioxidant activity measured in emulsion systems [26]. The different emulsifier evaluated was SDS, CTAB, Brij58 and PHLC, and their partitioning study revealed increased solubility effect of the emulsifiers in the following order: PHLC $<$ SDS $<$ Brij58 $<$ CTAB. The antioxidant activity of gallic acid and ethyl gallate based on the formation of hydroperoxides and hexanal increased in the following order: CTAB (no activity measured) $<$ Brij58 $<$ PHLC $<$ SDS. Gallic acid only showed antioxidant activity with PHLC stabilized emulsions. This was a reverse order compared to the partitioning measured. Hence, it was suggested that the increased partitioning into the emulsifier layer and lipid counteract the hydrogen-donating ability, and lower the activity of the antioxidants [24]. Moreover, Pekkarinen et al. [257] evaluated antioxidative effect and partitioning of phenolics in different systems. Interaction between caffeic acid and Tween 20 differed from other phenolics such as vanillic acid, ferulic acid and sinapic acid evaluated, since Tween 20 exhibited higher solubilisation capacity for caffeic acid than for other phenolic acids. Additionally, Pekkarinen et al. [257] concluded that these antioxidant-emulsifier interactions have a strong influence on the partitioning of antioxidants. The partitioning results obtained in this study confirmed that Citrem and Tween as emulsifiers results in differences in the partitioning of caffeic acid and caffeates. Less caffeic acid and caffeates were present in the aqueous phase when Tween was applied. This clearly demonstrated stronger antioxidant-emulsifier interaction with Tween compared to Citrem. Moreover, Citrem is an anionic emulsifier, thus, the interface is negatively charged and will repel negatively charged antioxidants i.e. caffeic acid, which could explain why caffeic acid interacted

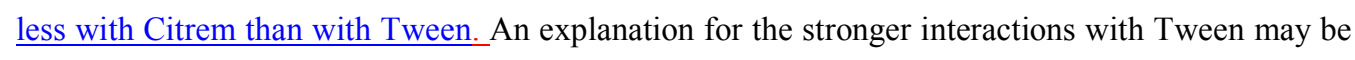
the molecular structure of the emulsifiers, since Tween is a larger and more bulky molecule than Citrem; however, this has to be further evaluated. Furthermore, Schwarz et al. [19, 26ㅁ, 27ㅁ] evaluated partitioning of different antioxidants in dispersed lipid systems with different emulsifiers. 
Significant differences were observed in partitioning of the antioxidants between phases, both as a function of $\mathrm{pH}$ and emulsifier type and concentration. It was concluded from the results that determination of antioxidant partitioning may be an important tool to select antioxidants structurally designed to localize at the surfaces [19], however, the partitioning of the antioxidants cannot alone explain the measured antioxidant activity in emulsions [279]. Results from another study by Schwarz et al. [2830] evaluating antioxidant activity of antioxidants with different lipophilicity in bulk oil, $\mathrm{e} / \mathrm{W} / \mathrm{O}$ and $w / \theta \underline{\mathrm{W} / \mathrm{O}}$ emulsions with different emulsifiers led to the assumption that differences in antioxidant activity for the same emulsion type might be additionally influenced by interaction with the emulsifier dominating the interfaces in the emulsion system [2830]. The obtained results for the two emulsifiers together with the partitioning study may also here lead to the assumption that emulsifier-antioxidant interactions (e.g. hydrogen bionding) affected the antioxidant activity of the caffeates. However, the type of interactions and the impact of antioxidant-emulsifier interactions on the radical scavenging activity have to be studied more in $\underline{\text { more details to make further conclusions. }}$

Impact of endogenous tocopherols on the efficacy of caffeic acid and caffeates. The presence of endogenous tocopherol not only changed the antioxidant activity of caffeic acid and caffeates in both the storage experiment and in the CAT assay, but also their partitioning in the emulsion system. A tendency to less caffeic acid and caffeates $(\mathrm{C} 1, \mathrm{C} 4$ and $\mathrm{C} 12)$ present in the aqueous phase with endogenous tocopherol in the emulsion system was observed. This may indicate some interactions between tocopherol and caffeic acid / caffeates both for the antioxidative effect and localization in the emulsion system. The use of a combination of antioxidants to produce synergistic interaction has been reported earlier e.g. tocopherol regeneration by ascorbic acid, polyphenols and flavonoids [2931-324]. Panaya et al. [324] carried out the only study investigating interactions between tocopherol and a phenol (rosmarinic acid) and its alkyl esters (rosmarinates, C4, C12 and 
C20) in Tween20 stabilized emulsions. Rosmarinic acid exhibited strongest synergistic interaction with tocopherol, and $\mathrm{C} 4$ and $\mathrm{C} 12$ esters exhibited small synergistic interaction. An antagonistic interaction was observed with $\mathrm{C} 20$ ester and tocopherol. Thus, the more hydrophilic rosmarinic acid exhibited more interactions with the tocopheryl radical than the esters. In the present study, the emulsion with the more hydrophilic caffeic acid exhibited better oxidative stability than the emulsions with the esters (more hydrophobic antioxidants) when tocopherol was present as also observed with rosmarinic acid and rosmarinates. Actually, caffeic acid turned from being prooxidative without tocopherol present to being the most efficient antioxidant with endogenous tocopherol present in Tween stabilized emulsion. In emulsions, the majority of the emulsifier is accumulated at the oil-water interface. However, a part of the emulsifier is not associated with the oil-water interface if the emulsifier concentration is above the CMC (critical micellar concentration, CMC Tween80 13-15 mg/L, Sigma) and will form micelles in the aqueous phase. In this study, the concentration of Tween was much higher than $\mathrm{CMC}(10 \mathrm{~g} / \mathrm{L})$. The decreased antioxidant efficiency of the different caffeates compared with caffeic acid is suggested to be due to the solubilisation of caffeates in Tween micelles, thus, localized away from the interface unable to inhibit lipid oxidation. Although, micelles are not isolated structures, they compromise structures that are in dynamic equilibrium with other structures in the emulsion system. This means that components can be exchanged between the different structures i.e. between micelles and emulsion droplets $[35,36]$. Thus, more studies are needed to further elucidate the differences observed in partitioning and antioxidant effect in the presence of endogenous tocopherols.

Additionally, it is assumed that caffeic acid is located in close proximity to the interface where it regenerates tocopherol at the interface in spite of the repelling effect of two negatively charged compounds (Citrem and caffeic acid). The proposed partial location of tocopherol at the interface is supported by Jacobsen et al. [37] who reported that ca. $6 \%$ of the alpha-tocopherol present in 
7 8518 9 $10^{519}$ $11^{520}$ $12^{520}$ 13 14521 15 $16^{522}$ 17 $18^{523}$ 19 $20^{524}$

$21_{525}$ 22

mayonnaise was located at the interface whereas the remaining tocopherol was located in the oil phase. Therefore, lipophilization is not needed to improve the oxidative stability of these emulsions, due to tocopherols location at the interface.

Antioxidant hypotheses and efficacies of caffeic acid and caffeates. The polar paradox was not confirmed in this study since caffeic acid was more efficient as antioxidant than caffeates in Citrem stabilized emulsions when endogenous tocopherol was present. In Tween stabilized emulsions with endogenous tocopherol caffeic acid followed by methyl caffeate were the most efficient antioxidants, whereas, caffeic acid acted as a prooxidant when tocopherol was not present. No cutoff effect was observed for the lipophilized caffeic acid, since the most efficient antioxidant in both Citrem and Tween stabilized emulsions when tocopherol was present was C0 (caffeic acid). A similar finding was observed for the CAT assay with endogenous tocopherol present. Without endogenous tocopherol present, the caffeates were most efficient antioxidants in Tween stabilized emulsions, a cut-off effect was found at C16. However, the efficiency of $\mathrm{C} 20$ was still an intermediate to strong antioxidant. The CAT assay showed a cut-off effect at around C8 and C12 with no endogenous tocopherol present. The partitioning experiment clearly showed an effect of the chain length, with less antioxidant present in the aqueous phase with increasing antioxidant lipophilicity. Pekkarinen et al. [247] observed that the proportion of antioxidant solubilized in the lipid phase and particularly in the interface did not necessarily reflect the efficiency of the antioxidant. It was assumed from their evaluation of antioxidant activity and partitioning that specific interactions of the antioxidant with other compounds e.g. emulsifiers, and intermolecular hydrogen bonds may play an important role in reducing antioxidant activity. Furthermore, antioxidant-emulsifier interaction has a strong influence on partitioning of the antioxidant. It is suggested that the caffeates interacts with the emulsifier in form of micelles when endogenous tocopherols are present, resulting in reduced or no antioxidant activity. When endogenous 
8542 tocopherols are not present the caffeates is more likely to be solubilized at the interface instead of 9 $10^{543}$

the tocopherols. However, this has to be evaluated more in depth to conclude further on these differences in partitioning and antioxidant activity with the impact of endogenous tocopherols.

Conclusions. Partitioning and antioxidant activity of caffeic acid and caffeates were influenced both by the emulsifier type and the presence of endogenous tocopherols. Thus, this study clearly demonstrated different emulsifier-antioxidant and antioxidant-antioxidant interactions that affected the efficacy of the evaluated caffeic acid and caffeates as antioxidant in emulsions. The hypotheses about antioxidant in emulsions are based on simple emulsions systems without the presence of tocopherols. However, the impact of the presence of tocopherols on the efficacy of other antioxidants is important since most food systems contain tocopherol. 


\section{Acknowledgements}

9 $10_{553}$ 11 12554 13 14555 15

16 $17^{556}$

$18_{557}$ 19 20558 21

22 $23^{55}$ 24 25 26 27 28

29

30

31

32

33

34

35

36

37

38

39

40

41

42

43

44

45

46

47

48

49

50

51

52

53

54

55

56

57

58

59

60

We thank Thi Thu Trang Vu for skillful work in the laboratory, Maritex Norway (subsidiary of TINE BA, Norway) and Danisco Ingredients (Dupont, Brabrand, Denmark) for providing the oils and Citrem, respectively.

The study is a part of the project entitled "Phenolipids as antioxidants in omega- 3 model and real food systems - Effect of alkyl chain length and concentration" with project no 10-093655 financed by the Danish Research Council, Technology and Production.

The authors declare no conflicts of interest. 


\section{Reference list}

1. McClements, D. J.; Decker, E. A. Lipid oxidation in oil-in-water emulsions: impact of molecular environment on chemical reactions in heterogeneous food systems. J. Food Sci., 2000, 65, 12701282.

2. Decker, E. A. Strategies for manipulating the prooxidative / antioxidative balance of foods to maximize oxidative stability. Trends in Food Sci. Technol., 1998, 9, 241-248.

3. Porter, W. L. Paradoxical behavior of antioxidants in food and biological systems. Toxicol. Ind. Health, 1993, 9, 93-122.

4. Laguerre, M.; Giraldo, L. J. L.; Lecomte, J.; Figueroa-Espinoza, M. C.; Barea, B.; Weiss, J.; Decker, E. A.; Villeneuve, P. Chain Length Affects Antioxidant Properties of Chlorogenate Esters in Emulsion: The Cutoff Theory Behind the Polar Paradox. J. Agric. Food Chem., 2009, 57, $11335-$ 11342.

5. Frankel, E. N.; Huang, S.-W.; Kanner, J.; German, B. Interfacial phenomena in the evaluation of antioxidants: bulk oils vs emulsions. J. Agric. Food Chem., 1994, 42, 1054-1059.

6. Laguerre, M.; Giraldo, L. J. L.; Lecomte, J.; Figueroa-Espinoza, M.-C.; Baréa, B.; Weiss, J.; Decker, E. A.; Villeneuve, P. Relationship between hydrophobicity and antioxidant ability of "phenolipids" in emulsion: a parabolic effect of the chain length of rosmarinate esters. J. Agric. Food Chem., 2010, 58, 2869-2876.

\section{Laguerre, M.; Sørensen, A.-D. M.; Bayrasy, C.; Lecomte, J.; Jacobsen, C.; Decker, E. A.;} Villeneuve, P. Role of hydrophobicity on antioxidant activity in lipid dispersions from the polar paradox to the cut-off theory. In: Lipid oxidation: challenges in food systems. Ed. Logan, A.; Nienaber, U.; Pan, X. AOCS Press, Urbana, IL. 2013 (p. 261-296) ISBN 9780983079163. 
8. Sørensen, A.-D. M.; Durand, E.; Laguerre, M.; Bayrasy, C.; Lecomte, J.; Villeneuve, P.; Jacobsen, C. Antioxidant properties and efficacies of synthesized alkyl caffeates, ferulates and coumarates. J. Agric. Food Chem., 2014, 62, 12553-12562.

9. Alemán, M.; Bou, R.; Guardiola, F.; Durand, E.; Villeneuve, P.; Jacobsen, C.; Sørensen, A.-D. M. Antioxidative effect of lipophilized caffeic acid in fish oil enriched mayonnaise and milk. Food Chem., 2015, 167, 236-244.

10. Panya, A.; Laguerre, M.; Bayrasy, C.; Lecomte, J.; Villeneuve, P.; McClements, D.J.; Decker, E. A. An investigation of the versatile antioxidant mechanisms of action of rosmarinate alkyl esters in oil-in-water emulsions. J. Agric. Food Chem., 2012, 60, 2692-2700.

11. Lee, J. H.; Panya, A.; Laguerre, M.; Bayrasy, C.; Lecomte, J.; Villeneuve, P.; Decker, E. A. Comparison of the antioxidant capacities of rosmarinate alkyl esters in riboflavin photosensitized oil-in-water emulsion. J. Am. Oil Chem. Soc., 2013, 90, 225-232.

12. Barden, L.; Barouh, N.; Villeneuve, P.; Decker, E. A. Impact of hydrophobicity on antioxidant efficacy in low-moisture food. J. Agric. Food Chem., 2015, 63, 5821-5827.

13. AOCS Official Method Ce 8-89. Determination of Tocopherols and Tocotrienols in Vegetable Oils and Fats by HPLC. Champaign, IL, USA, 1997.

14. Sørensen, A.-D. M.; Nielsen, N. S.; Yang, Z.; Xu, X.; Jacobsen, C. The effect of lipohilization of dihydrocaffeic acid on its antioxidative properties in fish-oil-enriched emulsion. Eur. J. Lipid Sci. Technol., 2012, 114, 134-145.

15. Rawle, A. Basic principles of particle size analysis. Malvern Instruments Ltd., 1996. 
16. Bligh, E. G.; Dyer, W. J. A rapid method of total lipid extraction and purification. Can. J. Biochem. Physiol., 1959, 37, 911-917.

17. Iverson, S. J.; Lang, S. L. C.; Cooper, M. H. Comparison of the bligh and dyer and folch methods for total lipid determination in broad range of marine tissue. Lipids, 2001, 36, 1283-1287.

18. Shantha, N. C.; Decker, E. A. Rapid, sensitive, iron-based spectrophotometric methods for determination of peroxide values of food lipids. J. AOAC Int., 1994, 77, 421-424.

19. Schwarz, K.; Frankel, E. N.; German, J. B. Partition behaviour of antioxidative phenolic compounds in heterophasic systems. Fett-Lipid 1996, 98, 115-121.

20. Sørensen, A.-D. M.; Nielsen, N. S.; Decker, E. A.; Let, M. B.; Xu, X.; Jacobsen, C. The efficacy of compounds with different polarities as antioxidant in emulsions with omega-3 lipids. J. Am. Oil Chem. Soc., 2011, 88, 489-502.

21. Laguerre, M.; Lopez-Giraldo, L. J.; Lecomte, J.; Barea, B.; Cambon, E.; Tchobo, P. F.; Barouh, N.; Villeneuve, P. Conjugated autoxidizable triene (CAT) assay: A novel spectrophotometric method for determination of antioxidant capacity using triacylglycerol as ultraviolet probe. Anal. Biochem., 2008, 380, 282-290.

22. Jacobsen, C.; Let, M. B.; Nielsen, N. S.; Meyer, A. S.; Antioxidant strategies for preventing oxidative flavor deterioration of food enriched with n-3 polyunsaturated lipids: a comparative evaluation. Trends Food Sci. Technol., 2008, 19, 76-93.

23. Sørensen, A.-D. M.; Haarh, A.-M.; Becker, E. M.; Skibsted, L. H.; Bergenståhl, B.; Nilsson, L.; Jacobsen, C. Interactions between iron, phenolic compounds, emulsifiers, and pH in omega-3enriched oil-in-water emulsions. J. Agric. Food Chem., 2008, 56, 1740-1750. 


\section{Costa, M.; Losada-Barreiro, S.; Paiva-Martins, F.; Bravo-Díaz, C.; Romsted, L. S. a direct correlation between the antioxidant efficiencies of caffeic acid and its alkyl esters and their concentrations in the interfacial region of olive oil emulsions. The pseudophase model interpretation of the "cut-off"effect. Food Chem., 2015, 175, 233-242. \\ 25. Costa, M.; Losada-Barreiro, S.; Paiva-Martins, F.; Bravo-Díaz, C. Optimizing the efficiency of antioxidants in emulsions by lipophilization: tuning interfacial concentrations. RSC Adv., 2016, 6, 91483-91493.}

24․․ Stöckman, H.; Schwarz, K.; Huynh-Ba, T. The influence of various emulsifiers on the partitioning and antioxidant activity of hydroxybenzoic acids and their derivatives in oil-in-water emulsions. J. Am. Oil Chem. Soc., 2000, 77, 535-542.

257. Pekkarinen, S.S.; Stöckman, H.; Schwarz, K.; Heinonen, I.M.; Hopia, A.I. Antioxidant activity and partitioning of phenolic acids in bulk and emulsified methyl linoleate. J. Agric. Food Chem., 1999, 41, 3036-3043.

26․․ Heins, A.; Garamus, V. M.; Steffen, B.; Stöckmann, H.; Schwarz, K. Impact of phenolic antioxidants on structural properties of micellar solutions. Food Biophys., 2006, 1, 189-201. 279. Oehlke, K.; Heins, A.; Stöckmann, H.; Schwarz, K. Impact of emulsifier microenvironments on acid-base equilibrium and activity of antioxidants. Food Chem., 2010, 118, 48-55.

2830. Schwarz, K.; Huang, S.-W.; German, B.; Tiersch, B.; Hartmann, J.; Frankel, E.N. Activities of antioxidants are affected by colloidal properties of oil-in-water and water-in-oil emulsions and bulk oils. J. Agric. Food Chem., 2000, 48, 4874-4882.

2931. Medina, I.; Undeland, I.; Larsson, K.; Storrø, I.; Rustad, T.; Jacobsen, C. Activity of caffeic acid in different fish lipid matrices: a review. Food chem., 2012, 131, 730-740. 
302. Laranjinha, J.; Vieiva, O.; Madeira, V.; Almeida, L. Two related phenolic antioxidants with opposite effects on vitamin E content in low density lipoproteins oxidized by ferrylmyoglobin: composition vs regeneration. Arch. Biochem. Biophys., 1995, 323, 373-381.

343. Iglesias, J.; Pazos, M.; Andersen, M. L.; Skibsted, L. H.; Medina, I. Caffeic acid as antioxidant in fish muscle: mechanism of synergism with endogenous ascorbic acid and alpha-tocopherol. J. Agric. Food Chem., 2009, 57, 675-681.

32․․ Panya, A.; Kittipongpittaya, K.; Laguerre, M.; Bayrasy, C.; Lecomte, J.; Villeneuve, P.; McClements, D.J.; Decker, E.A. Interactions between $\alpha$-tocopherol and rosmarinic acid and its alkyl esters in emulsions: synergistic, additive, or antagonistic effect? J. Agric. Food Chem., 2012, 60, 10320-10330.

35. Skhiri, Y.; Gruner, P.; Semin, B.; Brosseau, Q.; Pekin, D.; Mazutis, L.; Goust, V.;

Kleinschmidt, F.; Harrak, A. E.; Hutchison, J. B.; Mayot, E.; Bartolo, J.-F.; Griffiths, A. D.; Taly, $\underline{\text { V.; Baret, J.-C. Dynamics of molecular transport by surfactants in emulsions. Soft Matter, 2012, 8, }}$ $\underline{10618-10627 .}$

36. You, Y.; Bloomfield, A.; Liu, J.; Fu, L.; Herzon, S. B.; Yan, C. E. Real-time kinetics of

$\underline{\text { surfactant molecule transfer between emulsion particles probed by in situ second harmonic }}$ generation spectroscopy. J. Am. Oil Chem. Soc., 2012, 134, 4264-4268.

37. Jacobsen, C.; Schwarz, K.; Stoeckmann, H.; Meyer, A.S.; Adler-Nissen, J. Partitioning of selected antioxidants in mayonnaise. J. Agric. Food Chem., 1999, 47, 3601-3610. 


\section{$8664 \quad$ Figure legends}

9

Figure 1 PCA build on results obtained from PV, voltatiles (1-penten-3-one, 1-penten-3-ol, 4heptenal, 2,4-heptadienal, hexanal and nonanal) and $\alpha$-tocopherols measured on Citrem stabilized emulsions during storage (15 days) using full cross validation. A) Correlation loadings and B) Scores plot. Abbreviations for sample codes refer to Table 1.

Figure 2 PCA build on results obtained from PV, voltatiles (1-penten-3-one, 1-penten-3-ol and 2,4-

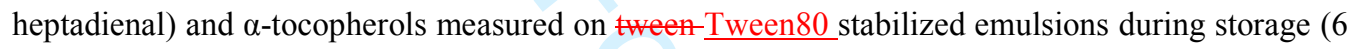
days) using full cross validation. A) Correlation loadings and B) Scores plot. Abbreviations for sample codes refer to Table 1 .

Figure 3 PCA build on results obtained from PV and voltatiles (1-penten-3-one, 1-penten-3-ol and

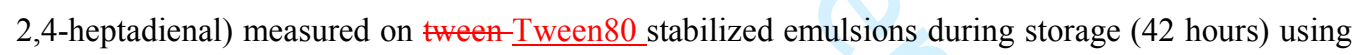
full cross validation. A) Correlation loadings and B) Scores plot. Abbreviations for sample codes refer to Table 1.

Figure 4 CAT Value of caffeic acid and caffeates (C1-C16) measured in the concentration range of $0.5-2 \mu \mathrm{M}$. $\bullet$ CAT Values determined without endogenous tocopherols (normal condition for the CAT assay, published in Sørensen et al. [8]) and o CAT Values determined with endogenous tocopherols (modified CAT assay). 
Table 1 Experimental design of experiment 1 and 2.

\begin{tabular}{|c|c|c|c|c|}
\hline Experiment & Sample code & Emulsifier & Oil & Antioxidant \\
\hline & C_Con & Citrem & FO & No antioxidant \\
\hline & C_CA C0 & Citrem & FO & Caffeic acid \\
\hline $\mathrm{E}$ & C_CA C1 & Citrem & FO & Methyl caffeate \\
\hline $\mathrm{X}$ & $\mathrm{C}_{-} \mathrm{CA} \mathrm{C} 4$ & Citrem & FO & Butyl caffeate \\
\hline \multirow[t]{2}{*}{$\mathrm{P}$} & C_CA C8 & Citrem & FO & Octyl caffeate \\
\hline & C_CA C12 & Citrem & FO & Dodecyl caffeate \\
\hline \multirow[t]{6}{*}{1} & C_CA C16 & Citrem & FO & Hexadecyl caffeate \\
\hline & $\mathrm{C}_{-} \mathrm{CA} \mathrm{C} 20$ & Citrem & FO & Eicosyl caffeate \\
\hline & T_Con & Tween80 & $\mathrm{FO} / \mathrm{RO}$ & No antioxidant \\
\hline & T_CA C0 & Tween 80 & $\mathrm{FO} / \mathrm{RO}$ & Caffeic acid \\
\hline & $\mathrm{T}_{-} \mathrm{CAC} 1$ & Tween80 & $\mathrm{FO} / \mathrm{RO}$ & Methyl caffeate \\
\hline & $\mathrm{T}_{-} \mathrm{CAC} 4$ & Tween 80 & $\mathrm{FO} / \mathrm{RO}$ & Butyl caffeate \\
\hline $\mathrm{E}$ & $\mathrm{T}_{-} \mathrm{CAC} 8$ & Tween 80 & $\mathrm{FO} / \mathrm{RO}$ & Octyl caffeate \\
\hline $\mathrm{X}$ & T_CA C12 & Tween 80 & $\mathrm{FO} / \mathrm{RO}$ & Dodecyl caffeate \\
\hline \multirow[t]{2}{*}{$\mathrm{P}$} & T_CA C16 & Tween80 & $\mathrm{FO} / \mathrm{RO}$ & Hexadecyl caffeate \\
\hline & TS_Con & Tween80 & $\mathrm{S} F O / \mathrm{RO}$ & No antioxidant \\
\hline \multirow[t]{7}{*}{2} & TS_CA C0 & Tween80 & $\mathrm{S} \mathrm{FO} / \mathrm{RO}$ & Caffeic acid \\
\hline & TS_CA C1 & Tween 80 & $\mathrm{~S} \mathrm{FO} / \mathrm{RO}$ & Methyl caffeate \\
\hline & TS_CA C4 & Tween80 & $\mathrm{S} \mathrm{FO} / \mathrm{RO}$ & Butyl caffeate \\
\hline & TS_CA C8 & Tween80 & $\mathrm{S} \mathrm{FO} / \mathrm{RO}$ & Octyl caffeate \\
\hline & TS_CA C12 & Tween 80 & $\mathrm{~S} \mathrm{FO/RO}$ & Dodecyl caffeate \\
\hline & TS_CA C16 & Tween80 & $\mathrm{S} \mathrm{FO} / \mathrm{RO}$ & Hexadecyl caffeate \\
\hline & TS_CA C20 & Tween 80 & $\mathrm{~S} \mathrm{FO} / \mathrm{RO}$ & Eicosyl caffeate \\
\hline
\end{tabular}

Abbreviations: FO Fish oil, FO/RO Fish oil and rapeseed oil (1:1, w/w) and S FO/RO Stripped fish oil and rapeseed oil $(1: 1, \mathrm{w} / \mathrm{w})$ 
686 Table 2 Concentration $[\mu \mathrm{M}]$ of caffeic acid and caffeates (Methyl, Butyl, Octyl and Dodecyl) measured in the aqueous phase of different

687 systems: 95\% Buffer / 5\% Oil, 99\% Buffer / 1\% Emulsifier and Emulsion (5\% Oil, 1\% Emulsifier and 94\% Buffer). Citrem and Tween80

688 were applied as emulsifier. Both non-stripped and stripped FO/RO was evaluated with Tween 80 as emulsifier.

\begin{tabular}{|c|c|c|c|c|c|c|c|c|}
\hline \multirow[b]{2}{*}{ Antioxidant } & \multicolumn{3}{|c|}{ Citrem and non-stripped oil } & \multicolumn{3}{|c|}{ Tween80 and non-stripped oil } & \multicolumn{2}{|c|}{ Tween80 and stripped oil } \\
\hline & Buffer / Oil & Buffer / Emulsifier & Emulsion & Buffer / Oil & $\begin{array}{c}\text { Buffer / } \\
\text { Emulsifier }\end{array}$ & Emulsion & Buffer / Oil & Emulsion \\
\hline CA CO & $101 \pm 3.1^{\mathrm{a}, \mathrm{b}, \mathrm{x}}$ & $91.5 \pm 3.6^{\mathrm{b}, \mathrm{x}}$ & $91.5 \pm 7.1^{\mathrm{b}, \mathrm{x}}$ & $93.7 \pm 11.4^{\mathrm{b}, \mathrm{x}}$ & $75.4 \pm 7.0^{\mathrm{c}, \mathrm{x}}$ & $74.0 \pm 4.3^{\mathrm{c}, \mathrm{x}}$ & $111 \pm 2.6^{\mathrm{a}, \mathrm{x}}$ & $78.4 \pm 10.1^{\mathrm{c}, \mathrm{x}}$ \\
\hline CA C1 & $86.2 \pm 0.9^{\mathrm{a}, \mathrm{y}}$ & $53.3 \pm 3.0^{\mathrm{b}, \mathrm{y}}$ & $43.8 \pm 8.2^{\mathrm{b}, \mathrm{y}}$ & $82.8 \pm 1.9^{\mathrm{a}, \mathrm{x}}$ & $15.4 \pm 3.3^{\mathrm{c}, y}$ & $10.1 \pm 2.5^{\mathrm{c}, \mathrm{y}}$ & $91.1 \pm 2.6^{\mathrm{a}, \mathrm{y}}$ & $15.3 \pm 3.1^{\mathrm{c}, \mathrm{y}}$ \\
\hline CA C4 & $11.4 \pm 0.4^{\mathrm{a}, \mathrm{b}, \mathrm{z}}$ & $4.09 \pm 0.6^{\mathrm{a}, \mathrm{b}, \mathrm{c}, \mathrm{z}}$ & $3.39 \pm 0.5^{\mathrm{b}, c, \mathrm{z}}$ & $11.3 \pm 0.7^{\mathrm{a}, \mathrm{b}, \mathrm{z}}$ & $2.10 \pm 0.3^{\mathrm{c}, \mathrm{z}}$ & $<$ detection & $13.0 \pm 1.1^{\mathrm{a}, \mathrm{z}}$ & $<$ detection \\
\hline CA C8 & $<$ detection & $<$ detection & $<$ detection & $<$ detection & $2.60 \pm 3.6^{\mathrm{z}}$ & $<$ detection & $<$ detection & $<$ detection \\
\hline CA C12 & $<$ detection & $<$ detection & $<$ detection & $2.00 \pm 0.6^{\mathrm{b}, \mathrm{z}}$ & $1.10 \pm 0.4^{\mathrm{z}}$ & $<$ detection & $9.70 \pm 4.2^{\mathrm{a}, \mathrm{z}}$ & $1.10 \pm 0.2^{\mathrm{b}, \mathrm{y}}$ \\
\hline
\end{tabular}

689 Different letters in superscript indicate significant differences. Significant differences within a row i.e. same antioxidant but different systems are denoted with a,b and

690 c, whereas significant differences within a column i.e. same system but different antioxidant are denoted with $\mathrm{x}, \mathrm{y}$ and $\mathrm{z}$. 
691 Table 3 Calculated inhibition percentages for selected lipid oxidation parameters (PV, 1-penten-3-ol, 1-penten-3-one and 2,4-

692 heptadienalA) measured at selected time points in the different emulsions. Citrem stabilized emulsions are reported at day 6 and 15 , Tween

693 stabilized emulsions with endogenous tocopherols at day 6 and Tween stabilized emulsions without endogenous tocopherols at 42 hours.

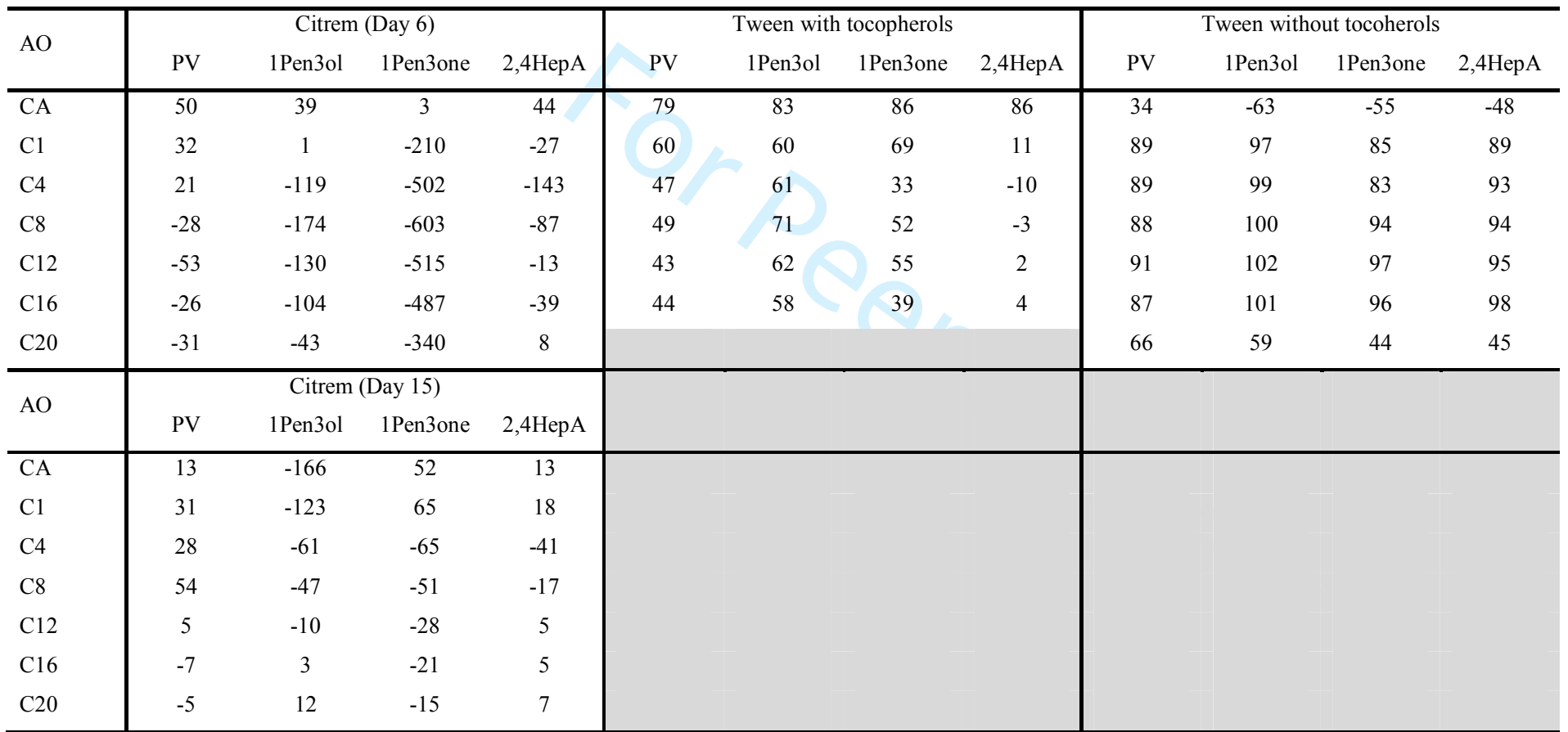

694 Abbreviation: AO Antioxidant; PV Peroxidae $*$ Value; 1Pen3ol 1-Penten-3-ol; 1Pen3one 1-Penten-3-one; 2,4HepA 2,4-HeptadienalA. The 2,4-heptadienal external 
A
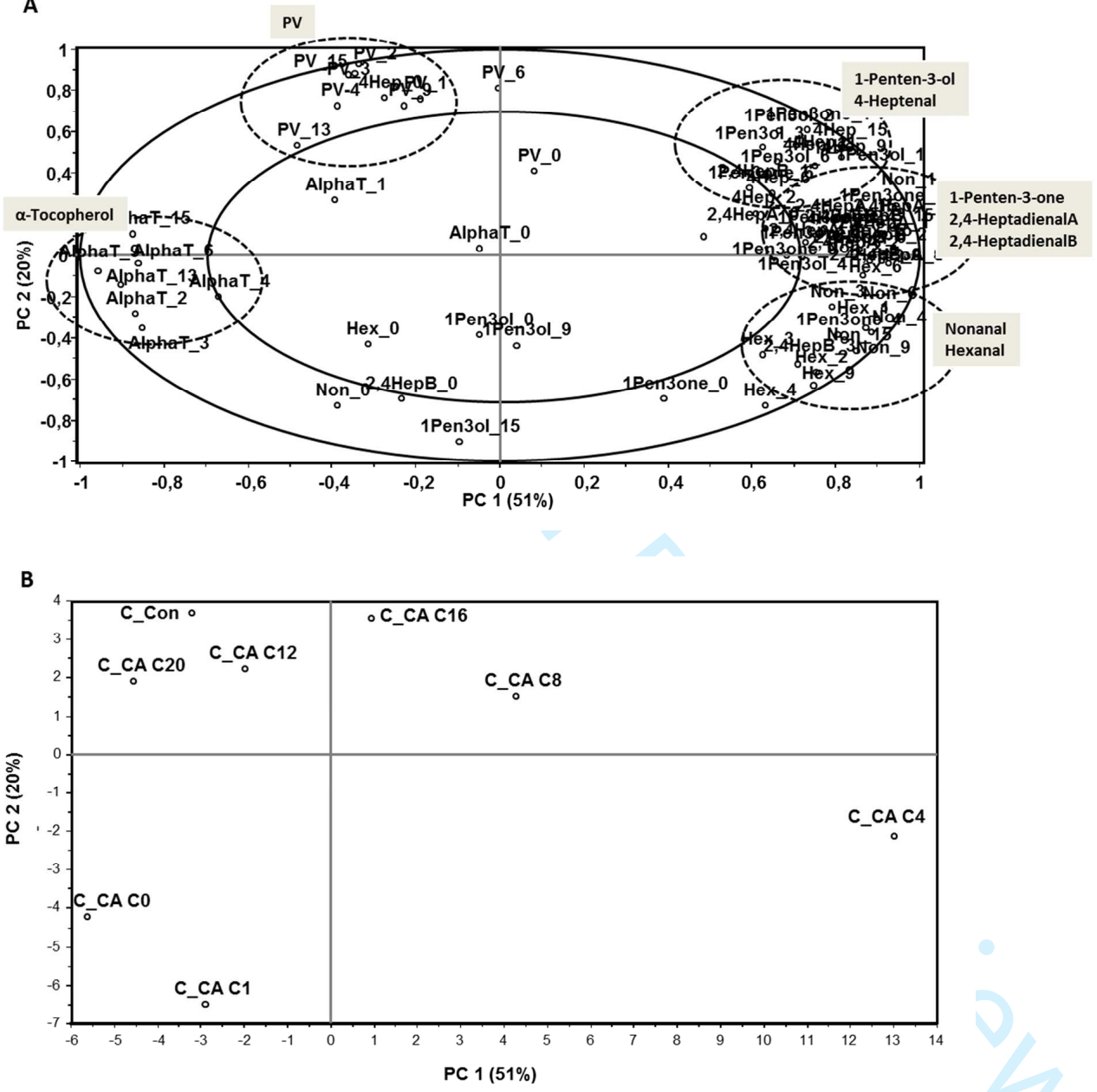

41700 


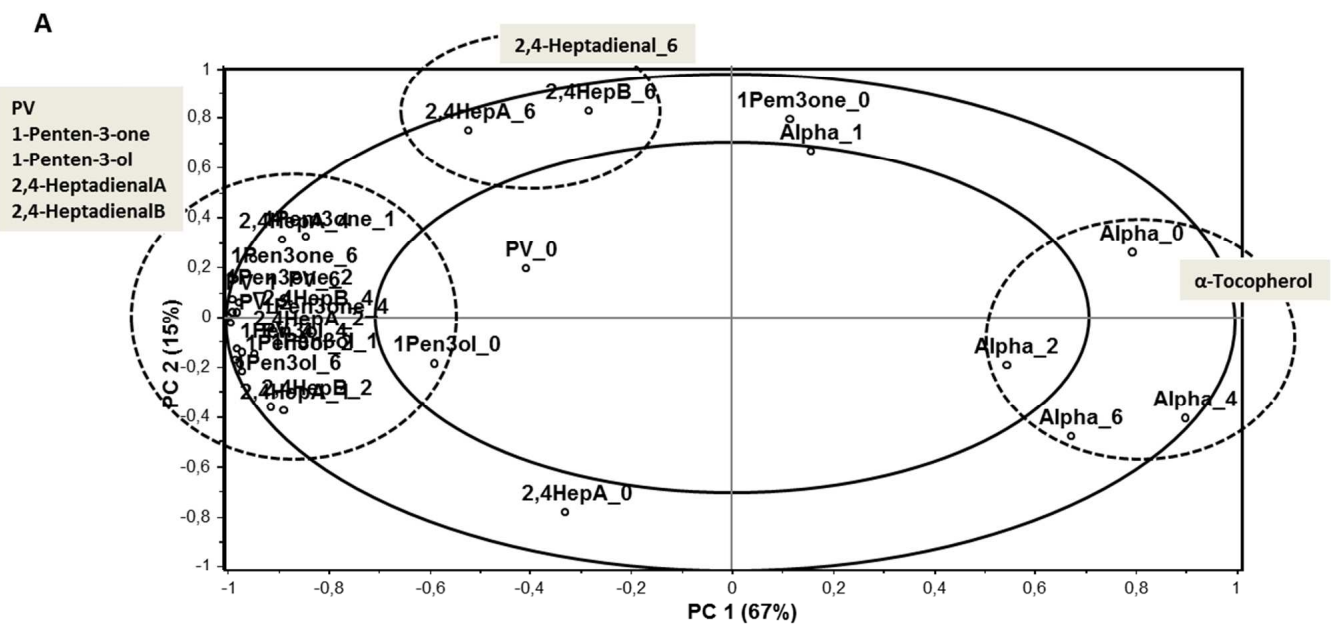

B

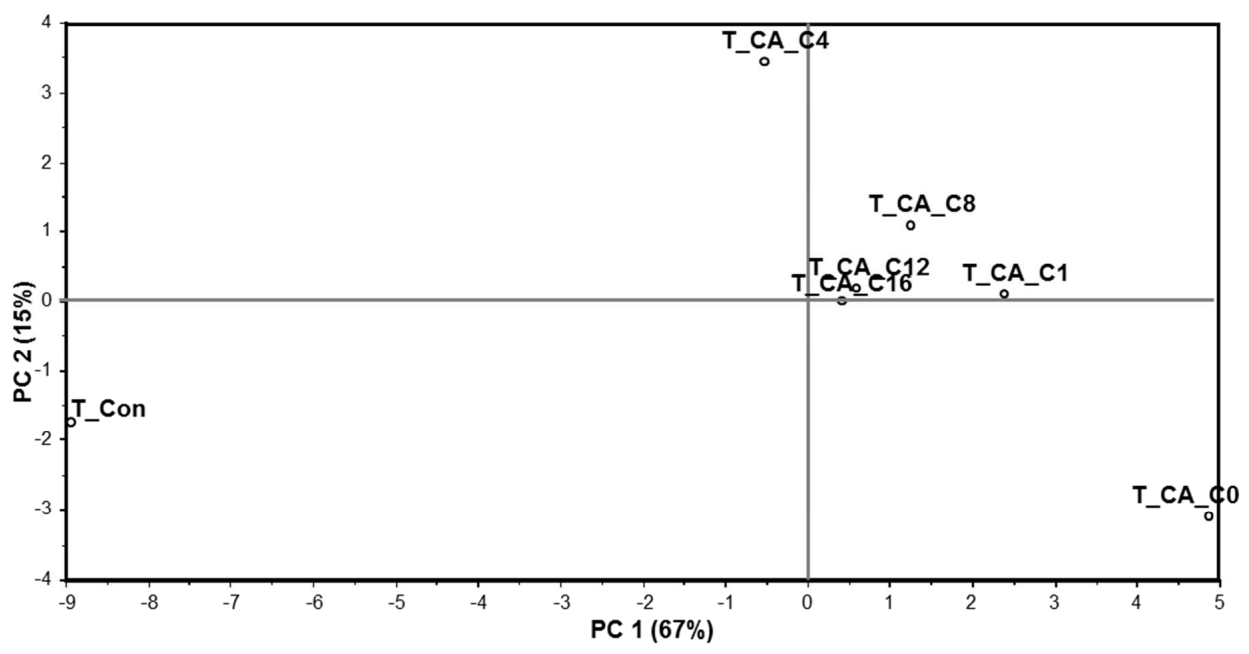

Figure 2 

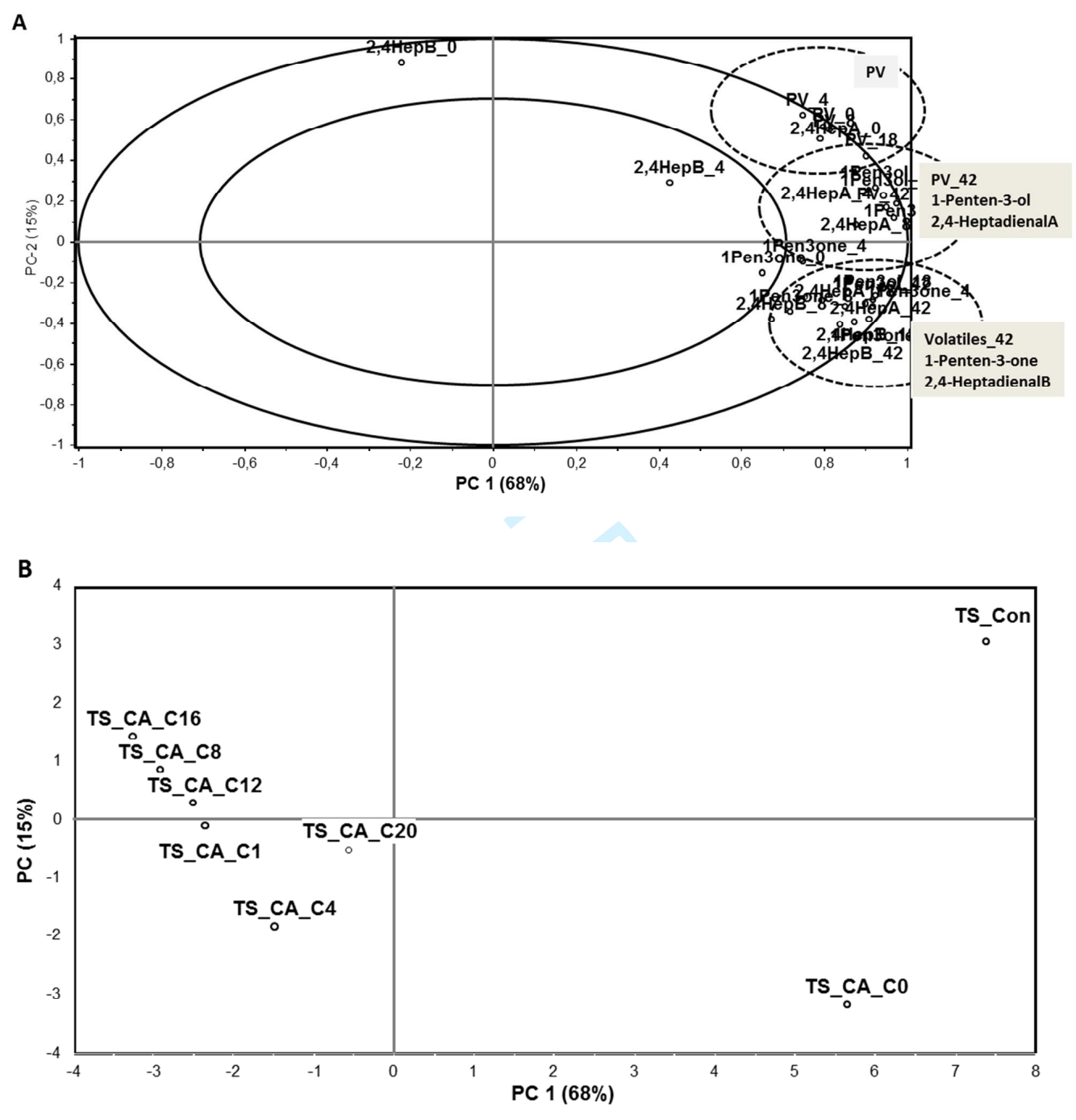

Figure 3 


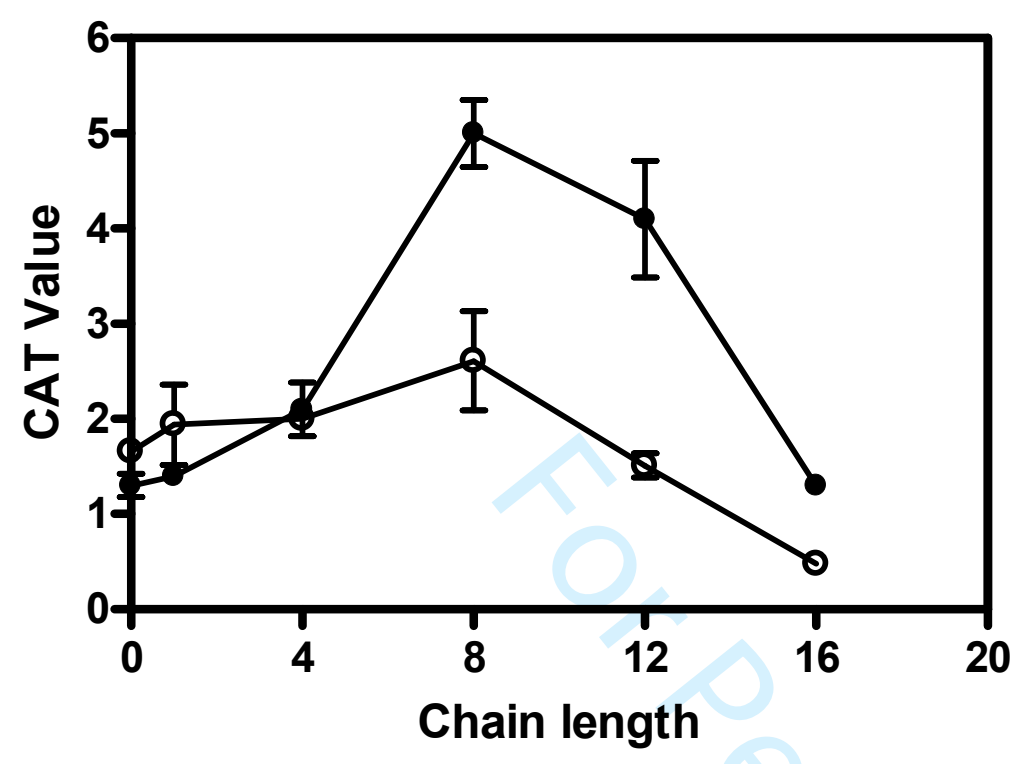


Graphical abstract. CAT Value determined in oil-in-water micro emulsions with caffeic acid and caffeates $(100 \mu \mathrm{M})$ and with and without endogenous tocopherol and. Lipid oxidation was initiated with the water soluble radical AAPH. 


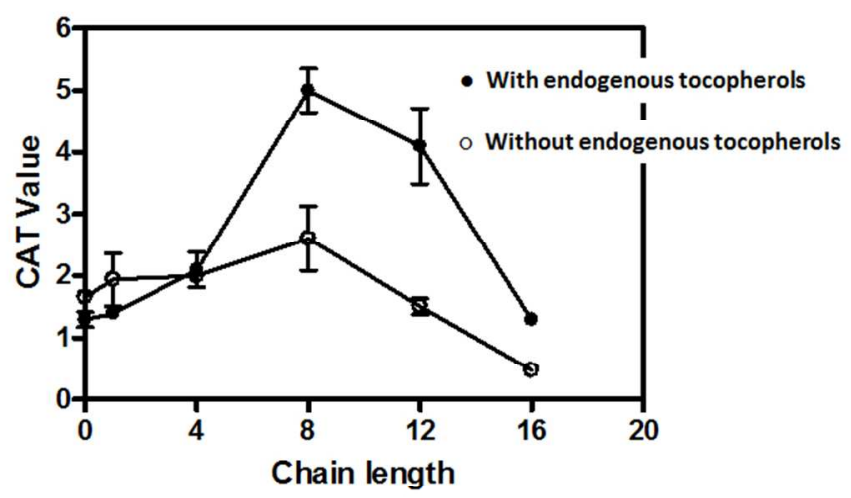

$254 \times 142 \mathrm{~mm}(96 \times 96 \mathrm{DPI})$

Wiley-VCH 